\title{
Lepton flavor violation in the Standard Model with general dimension-six operators
}

\section{A. Crivellin, ${ }^{a}$ S. Najjari ${ }^{b}$ and J. Rosiek ${ }^{b}$}

\author{
${ }^{a}$ Albert Einstein Center for Fundamental Physics, \\ Institute for Theoretical Physics, University of Bern, \\ Bern, Switzerland \\ ${ }^{b}$ Institute of Theoretical Physics, Department of Physics, University of Warsaw, \\ Warsaw, Poland \\ E-mail: crivellin@itp.unibe.ch, saereh.najjari@fuw.edu.pl, \\ janusz.rosiek@fuw.edu.pl
}

ABSTRACT: We study lepton flavor observables in the Standard Model (SM) extended with all dimension-6 operators which are invariant under the SM gauge group. We calculate the complete one-loop predictions to the radiative lepton decays $\mu \rightarrow e \gamma, \tau \rightarrow \mu \gamma$ and $\tau \rightarrow e \gamma$ as well as to the closely related anomalous magnetic moments and electric dipole moments of charged leptons, taking into account all dimension- 6 operators which can generate lepton flavor violation. Also the 3-body flavor violating charged lepton decays $\tau^{ \pm} \rightarrow \mu^{ \pm} \mu^{+} \mu^{-}$, $\tau^{ \pm} \rightarrow e^{ \pm} e^{+} e^{-}, \tau^{ \pm} \rightarrow e^{ \pm} \mu^{+} \mu^{-}, \tau^{ \pm} \rightarrow \mu^{ \pm} e^{+} e^{-}, \tau^{ \pm} \rightarrow e^{\mp} \mu^{ \pm} \mu^{ \pm}, \tau^{ \pm} \rightarrow \mu^{\mp} e^{ \pm} e^{ \pm}$and $\mu^{ \pm} \rightarrow e^{ \pm} e^{+} e^{-}$and the $Z^{0}$ decays $Z^{0} \rightarrow \ell_{i}^{+} \ell_{j}^{-}$are considered, taking into account all tree-level contributions.

Keywords: Rare Decays, Beyond Standard Model

ARXIV EPRINT: 1312.0634 


\section{Contents}

1 Introduction 1

2 The lepton flavor violating operators of dimension-6 4

$\begin{array}{lll}3 & \text { Observables related to the effective lepton-photon coupling } & 7\end{array}$

$\begin{array}{ll}3.1 & \text { Radiative lepton decays } \\ & 7.2\end{array}$

3.2 Anomalous magnetic moments and electric dipole moments 11

$4 \quad \ell_{i} \rightarrow \ell_{j} \ell_{k} \bar{\ell}_{l}$ decay rate $\quad 12$

4.1 Decay $\ell_{i} \rightarrow \ell_{j} \ell_{j} \bar{\ell}_{j} \quad 14$

$\begin{array}{lll}4.2 & \text { Decay } \ell_{i} \rightarrow \ell_{j} \ell_{k} \bar{\ell}_{k} & 15\end{array}$

$\begin{array}{lll}4.3 & \text { Decay } \ell_{i}^{ \pm} \rightarrow \bar{\ell}_{j}^{\mp} \ell_{k}^{ \pm} \ell_{k}^{ \pm} & 15\end{array}$

5 Lepton flavor violating $Z^{0}$ decays $\quad 15$

6 Numerical analysis $\quad 16$

$\begin{array}{llr}7 & \text { Conclusions } & 20\end{array}$

$\begin{array}{ll}\text { A Feynman rules and vector/scalar form-factors } & \mathbf{2 1}\end{array}$

A.1 Feynman rules involving gauge and Goldstone bosons 21

A.2 Feynman rules for 4-fermion operators 24

A.3 Vector and scalar form-factors contributing to off-shell $\ell_{i} \rightarrow \ell_{f} \gamma^{*}$ amplitude 25

\section{Introduction}

The Standard Model (SM) of strong and electroweak interactions has been successfully tested to a great precision [1]. Nevertheless, it is commonly accepted that it constitutes only an effective theory which is valid up an energy scale $\Lambda$ where new physics (NP) enters and additional dynamic degrees of freedom become important. A renormalizable quantum field theory valid above this scale should satisfy the following requirements:

(i) Its gauge group must contain the $\mathrm{SM}$ gauge group $\mathrm{SU}(3)_{C} \times \mathrm{SU}(2)_{L} \times \mathrm{U}(1)_{Y}$.

(ii) All SM degrees of freedom should be incorporated either as fundamental or as composite fields.

(iii) At low-energies it should reduce to the SM provided no undiscovered weakly coupled light particles exist (like axions or sterile neutrinos). 
In most theories of physics beyond the SM that have been considered, the SM is recovered at low energies via the decoupling of the heavy particles with masses of the order of $\Lambda \gg M_{Z}$. That such a decoupling at the perturbative level is possible in a renormalization quantum field theory is guaranteed by the Appelquist-Carazzone decoupling theorem [2]. This leads to the appearance of higher-dimensional operators which are suppressed by powers of $\Lambda$ and are added to the SM Lagrangian:

$$
\mathcal{L}_{\mathrm{SM}}=\mathcal{L}_{\mathrm{SM}}^{(4)}+\frac{1}{\Lambda} \sum_{k} C_{k}^{(5)} Q_{k}^{(5)}+\frac{1}{\Lambda^{2}} \sum_{k} C_{k}^{(6)} Q_{k}^{(6)}+\mathcal{O}\left(\frac{1}{\Lambda^{3}}\right)
$$

Here $\mathcal{L}_{\mathrm{SM}}^{(4)}$ is the usual renormalizable part of the SM Lagrangian which contains dimension2 and dimension- 4 operators only. $Q_{k}^{(5)}$ is the Weinberg operator giving rise to neutrino masses, $Q_{k}^{(6)}$ denote dimension-6 operators, and $C_{k}^{(n)}$ stand for the corresponding dimensionless coupling constants, i.e. the Wilson coefficients.

Even if the ultimate theory of NP at some high energy scale is not a quantum field theory, at low energies the effective theory still reduces to a quantum field theory [3] and it is possible to parametrize its effects at the electroweak scale in terms of these operators and the associated Wilson coefficients. Thus, one can search for NP in a model independent way by studying the SM extended with gauge invariant effective higher dimensional operators. Later, once a specific model is chosen, the Wilson coefficients can be calculated as a function of model parameters by matching the model of NP under consideration on the SM extended with such higher dimensional operators and one can calculate bounds on the specific model as well.

Flavor observables, especially flavor changing neutral current (FCNC) processes are an excellent probe of new physics since they are suppressed in the SM and therefore sensitive even to small NP contributions. This also means that these processes can stringently constrain the Wilson coefficients of the dimension- 6 operators induced by NP.

Especially the search for lepton flavor violation (LFV) is very promising since in the SM (extended with massive neutrinos) all flavor violating effects in the charged lepton sector are proportional to the very small neutrino masses - e.g. the decay rates of heavy charged leptons into lighter ones are suppressed by the ratio $m_{\nu}^{2} / M_{W}^{2}$ and thus are by far too small to be measurable in any foreseeable experiment. This in turn means that any observation of LFV would prove the existence of physics beyond the SM. In addition, LFV processes have the advantage of being "theoretically clean", i.e. they can be computed precisely without problems with non-perturbative QCD effects affecting similar observables in the quark sector.

Also the current experimental situation and prospects for the search for charged lepton flavor violation are very promising. In tables 1 and 2 we list the experimental bounds on the radiative lepton decays $\ell_{i} \rightarrow \ell_{f} \gamma$ and on the three-body lepton decays $\ell_{i} \rightarrow \ell_{j} \ell_{k} \ell_{l}$, respectively. Especially the limits on $\mu \rightarrow e$ transitions are very stringent due to constraints from the MEG and SINDRUM collaborations at the PSI and will be even further improved in the future: MEG can measure $\operatorname{Br}[\mu \rightarrow e \gamma]$ down to $6 \times 10^{-14}$ and a MEG upgrade [4] could increase the sensitivity by another order of magnitude. Furthermore, the electric dipole 


\begin{tabular}{|c|c|}
\hline Process & Experimental bound \\
\hline \hline $\operatorname{Br}[\tau \rightarrow \mu \gamma]$ & $4.4 \times 10^{-8}[5,6]$ \\
\hline $\operatorname{Br}[\tau \rightarrow e \gamma]$ & $3.3 \times 10^{-8}[5]$ \\
\hline $\operatorname{Br}[\mu \rightarrow e \gamma]$ & $5.7 \times 10^{-13}[7]$ \\
\hline
\end{tabular}

Table 1. Experimental upper limits on the branching ratios of the radiative lepton decays.

\begin{tabular}{|c|c|}
\hline Process & Experimental bound \\
\hline \hline $\operatorname{Br}\left[\tau^{-} \rightarrow \mu^{-} \mu^{+} \mu^{-}\right]$ & $2.1 \times 10^{-8}[8]$ \\
\hline $\operatorname{Br}\left[\tau^{-} \rightarrow e^{-} e^{+} e^{-}\right]$ & $2.7 \times 10^{-8}[8]$ \\
\hline $\operatorname{Br}\left[\tau^{-} \rightarrow e^{-} \mu^{+} \mu^{-}\right]$ & $2.7 \times 10^{-8}[8]$ \\
\hline $\operatorname{Br}\left[\tau^{-} \rightarrow \mu^{-} e^{+} \mu^{-}\right]$ & $1.7 \times 10^{-8}[8]$ \\
\hline $\operatorname{Br}\left[\mu^{-} \rightarrow e^{-} e^{+} e^{-}\right]$ & $1.0 \times 10^{-12}[9]$ \\
\hline
\end{tabular}

Table 2. Experimental upper limits on the branching ratios of the three body charged lepton decays.

\begin{tabular}{|c|c|c|c|}
\hline EDM & $\left|d_{e}\right|$ & $\left|d_{\mu}\right|$ & $d_{\tau}$ \\
\hline Bound $[\mathrm{e} \mathrm{cm}]$ & $8.7 \times 10^{-29}[10]$ & $1.9 \times 10^{-19}[11]$ & {$[-2.5,0.8] \times 10^{-17}[12]$} \\
\hline
\end{tabular}

Table 3. Experimental upper bounds (or allowed range for $d_{\tau}$ ) on electric dipole moments of the charged leptons.

moments (EDM) and the anomalous magnetic moments of charged leptons are theoretically closely related to $\ell_{i} \rightarrow \ell_{f} \gamma$ transitions and also here the experimental accuracies are very good, leading to strong upper bounds for the EDMs (see table 3). In addition, there is a longstanding discrepancy between the SM prediction and the measurement of the anomalous magnetic moment of the muon, which might be a hint for physics beyond the SM.

Lepton flavor violating processes have been studied in great detail in many specific extensions of the SM. For example in the MSSM non-vanishing decay widths for LFV processes are generated by flavor non-diagonal SUSY breaking terms [13-17]. Also extending the MSSM with right-handed neutrinos by the seesaw mechanism [18] gives rise to LFV [1927], as well as allowing for R-parity violation [28-30]. Other models like the littlest Higgs Model with T-Parity [31], two-Higgs-doublet models with generic flavor structures [32-35] or models with an extended fermion sector [36] have sources of lepton flavor violation, too. In order to make models of New Physics consistent with the non-observation of LFV processes in Nature, the assumption of Minimal Flavor Violation [37] has been extended to the lepton sector (see e.g. [38, 39]). Flavor changing $\tau$ decays have been studied in ref. [40] in a model independent way taking into account a (reducible) set of four-lepton operators and the magnetic lepton operators. However, a detailed model independent analysis with all gauge invariant operators is still pending. ${ }^{1}$

\footnotetext{
${ }^{1}$ For a model independent analysis for the Higgs sector of the SM see ref. [41, 42] and for anomalous top couplings ref. [41, 43].
} 
In this article we perform such a model independent analysis by considering the SM extended with all dimension-6 operators giving rise to lepton flavor violation which are invariant under the SM gauge group. We study the radiative lepton decays $\ell_{i} \rightarrow \ell_{f} \gamma$ and three-body charged lepton decays $\ell_{i} \rightarrow \ell_{j} \ell_{k} \ell_{l}$, as well as the anomalous magnetic moments and EDMs of charged leptons and the flavor violating $Z^{0} \rightarrow \ell_{i}^{-} \ell_{j}^{+}$decays.

It is worth noting that analyzing the LFV processes using the gauge-invariant basis of dimension- 6 operators automatically assures that the final results are also gauge invariant and contain all relevant contributions. Otherwise, one risks including just subset of diagrams contributing to a given process. For example it is quite common in the literature to calculate in a model of NP only the effective flavor changing $Z^{0}$-boson coupling to charged leptons and neglect the corrections to $W$ couplings, as the latter do not contribute at the tree-level to neutral current processes. However, both $Z^{0}$ and $W$ (and also Goldstone boson) couplings come from the same set of gauge-invariant higher-order operators, and are thus of the same size. In fact, (as our calculation shows explicitly) their contributions at least to some processes, like e.g. $\ell_{i} \rightarrow \ell_{f} \gamma$, are equally important and should be always considered together.

The outline of this article is as follows: after recalling the relevant dimension- 6 operators in the next section we will consider radiative lepton decays in section 3 (including the related anomalous magnetic moments and electric dipole moments of charged leptons), three-body charged lepton decays in section 4 and the flavor changing $Z^{0}$ decays, $Z^{0} \rightarrow \ell_{i}^{-} \ell_{j}^{+}$, in section 5. We calculate the full one-loop predictions for the $\ell_{i} \rightarrow \ell_{f} \gamma$ decays and all tree-level contributions for $\ell_{i} \rightarrow \ell_{j} \ell_{k} \ell_{l}$ decays in terms of the Wilson coefficients of the dimension- 6 operators. Section 6 deals with the numerical evaluation of our results and finally we conclude in section 7. An appendix summarizes the Feynman rules arising from the dimension- 6 operators after electroweak symmetry breaking and the additional form-factors for $\ell_{i} \rightarrow \ell_{f} \gamma^{*}$ amplitude for the case of an off-shell photon.

\section{The lepton flavor violating operators of dimension-6}

The complete (but still reducible) list of independent operators of dimension-5 and dimension- 6 which can be constructed out of SM fields and which are invariant under the SM gauge group fields was first derived in ref. [44]. In this article we follow the notation ref. [45] where the operator basis of ref. [44] was reduced to a minimal set. For completeness, we list below again the operators relevant for our discussion. We use the following indices and symbols:

- $a, b=1,2$ label the components of the weak isospin doublets.

- $i, j, k, l$ are flavor indices running from 1 to 3 .

- $L$ and $R$ stand for the chiralities.

- $\ell_{i}=\left(\begin{array}{c}\nu_{L i} \\ \ell_{L i}\end{array}\right)$ and $q_{i}=\left(\begin{array}{c}u_{L i} \\ d_{L i}\end{array}\right)$ stand for the lepton and the quark doublets. 


\begin{tabular}{|c|ccccc|c|}
\hline & \multicolumn{5}{|c|}{ fermions } & scalars \\
\hline field & $\ell_{L i}^{a}$ & $e_{R i}$ & $q_{L i}^{a}$ & $u_{R i}$ & $d_{R i}$ & $\varphi^{a}$ \\
\hline hypercharge $Y$ & $-\frac{1}{2}$ & -1 & $\frac{1}{6}$ & $\frac{2}{3}$ & $-\frac{1}{3}$ & $\frac{1}{2}$ \\
\hline
\end{tabular}

Table 4. Our conventions for the hypercharges of the SM fields.

- $e_{i}=\ell_{R i}, u_{i}=u_{R i}$ and $d_{i}=d_{R i}$ are the right-handed isospin singlets.

- $\varphi^{a}$ is the SM Higgs doublet where $\varphi^{2}$ is the neutral component.

The hypercharges of the SM fields are summarized in table 4. The sign convention for the covariant derivatives is

$$
\left(D_{\mu} \ell\right)^{a}=\left(\delta_{a b} \partial_{\mu}+\frac{1}{2} i g \tau_{a b}^{I} W_{\mu}^{I}+i g^{\prime} Y_{\ell} \delta_{a b} B_{\mu}\right) \ell^{b} .
$$

with $\tau^{I}$ being the Pauli matrices. The hermitian derivative terms are $\left(\varphi^{\dagger} \overleftarrow{D_{\mu}} \varphi \equiv\left(D_{\mu} \varphi\right)^{\dagger} \varphi\right)$ :

$$
\varphi^{\dagger} i \stackrel{\leftrightarrow}{D}_{\mu} \varphi \equiv i \varphi^{\dagger}\left(D_{\mu}-\overleftarrow{D}_{\mu}\right) \varphi \quad \text { and } \quad \varphi^{\dagger} i \stackrel{\leftrightarrow}{D_{\mu}^{I}} \varphi \equiv i \varphi^{\dagger}\left(\tau^{I} D_{\mu}-\overleftarrow{D}_{\mu} \tau^{I}\right) \varphi
$$

The gauge field strength tensors read

$$
\begin{aligned}
W_{\mu \nu}^{I} & =\partial_{\mu} W_{\nu}^{I}-\partial_{\nu} W_{\mu}^{I}-g \varepsilon^{I J K} W_{\mu}^{J} W_{\nu}^{K}, \\
B_{\mu \nu} & =\partial_{\mu} B_{\nu}-\partial_{\nu} B_{\mu} .
\end{aligned}
$$

In general, the SM can be extended by higher dimensional operators starting from dimension-5. However, there is just a single dimension-5 term respecting the SM gauge symmetry which, after electroweak symmetry breaking, generates neutrino masses and mixing angles - the Weinberg operator ( $C$ is the charge conjugation matrix and $\left.\varepsilon_{12}=+1\right)$ :

$$
Q_{\nu \nu}=\varepsilon_{a b} \varepsilon_{c d} \varphi^{a} \varphi^{c}\left(\ell_{i}^{b}\right)^{T} C \ell_{j}^{d} .
$$

This operator does not contribute directly (other then modifying the $U_{P M N S}$ matrix) to LFV processes in the charged lepton sector, consequently we do not consider it in the rest of the paper.

In table 5 we collect the independent dimension- 6 operators relevant for our discussion, i.e. all operators which can contribute to LFV processes in the charged lepton sector at the tree-level or at the 1-loop level. We neglect the operators which could give LFV effects only via the interference with the dimension-4 SM vertices containing the PMNS matrix, since such effects are suppressed by the small neutrino masses which we assume to be zero. The names of operators in the left column of each block should be supplemented with generation indices of the fermion fields whenever necessary, e.g. $Q_{\ell q}^{(1)} \rightarrow Q_{\ell q}^{(1) i j k l}$. Dirac and color indices (not displayed) are always contracted within the brackets. The same is true for the isospin indices, except for $Q_{\text {lequ }}^{(1)}$ and $Q_{\text {lequ }}^{(3)}$.

Note that different flavor index combinations of the 4-lepton operators can correspond to the same operator (for example $Q_{\ell \ell}^{i j k l}=Q_{\ell \ell}^{i l k j}=Q_{\ell \ell}^{k j i l}=Q_{\ell \ell}^{k l i j}$ ). For this reason, in the 


\begin{tabular}{|l|c||c|c||c|c|}
\hline \multicolumn{2}{|c||}{$\ell \ell \ell \ell$} & \multicolumn{2}{|c||}{$\ell \ell X \varphi$} & \multicolumn{2}{|c|}{$\ell \ell \varphi^{2} D$ and $\ell \ell \varphi^{3}$} \\
\hline$Q_{\ell \ell}$ & $\left(\bar{\ell}_{i} \gamma_{\mu} \ell_{j}\right)\left(\bar{\ell}_{k} \gamma^{\mu} \ell_{l}\right)$ & $Q_{e W}$ & $\left(\bar{\ell}_{o} \sigma^{\mu \nu} e_{j}\right) \tau^{I} \varphi W_{\mu \nu}^{I}$ & $Q_{\varphi \ell}^{(1)}$ & $\left(\varphi^{\dagger} i \stackrel{\leftrightarrow}{D_{\mu}} \varphi\right)\left(\bar{\ell}_{i} \gamma^{\mu} \ell_{j}\right)$ \\
$Q_{e e}$ & $\left(\bar{e}_{i} \gamma_{\mu} e_{j}\right)\left(\bar{e}_{k} \gamma^{\mu} e_{l}\right)$ & $Q_{e B}$ & $\left(\bar{\ell}_{i} \sigma^{\mu \nu} e_{j}\right) \varphi B_{\mu \nu}$ & $Q_{\varphi \ell}^{(3)}$ & $\left(\varphi^{\dagger} i \stackrel{\leftrightarrow}{D_{\mu}^{I}} \varphi\right)\left(\bar{\ell}_{i} \tau^{I} \gamma^{\mu} \ell_{j}\right)$ \\
$Q_{\ell e}$ & $\left(\bar{\ell}_{i} \gamma_{\mu} \ell_{j}\right)\left(\bar{e}_{k} \gamma^{\mu} e_{l}\right)$ & & & $Q_{\varphi e}$ & $\left(\varphi^{\dagger} i \stackrel{D_{\mu}}{\mu}\right)\left(\bar{e}_{i} \gamma^{\mu} e_{j}\right)$ \\
& & & $Q_{e \varphi 3}$ & $\left(\varphi^{\dagger} \varphi\right)\left(\bar{\ell}_{i} e_{j} \varphi\right)$ \\
\hline$Q_{\ell q}^{(1)}$ & $\left(\bar{\ell}_{i} \gamma_{\mu} \ell_{j}\right)\left(\bar{q}_{k} \gamma^{\mu} q_{l}\right)$ & $Q_{\ell d}$ & $\left(\bar{\ell}_{i} \gamma_{\mu} \ell_{j}\right)\left(\bar{d}_{k} \gamma^{\mu} d_{l}\right)$ & $Q_{\ell u}$ & $\left(\bar{\ell}_{i} \gamma_{\mu} l_{j}\right)\left(\bar{u}_{k} \gamma^{\mu} u_{l}\right)$ \\
$Q_{\ell q}^{(3)}$ & $\left(\bar{\ell}_{i} \gamma_{\mu} \tau^{I} \ell_{j}\right)\left(\bar{q}_{k} \gamma^{\mu} \tau^{I} q_{l}\right)$ & $Q_{e d}$ & $\left(\bar{e}_{i} \gamma_{\mu} e_{j}\right)\left(\bar{d}_{k} \gamma^{\mu} d_{l}\right)$ & $Q_{e u}$ & $\left(\bar{e}_{i} \gamma_{\mu} e_{j}\right)\left(\bar{u}_{k} \gamma^{\mu} u_{l}\right)$ \\
$Q_{e q}$ & $\left(\bar{e}_{i} \gamma^{\mu} e_{j}\right)\left(\bar{q}_{k} \gamma_{\mu} q_{l}\right)$ & $Q_{\ell e d q}$ & $\left(\bar{\ell}_{i}^{a} e_{j}\right)\left(\bar{d}_{k} q_{l}^{a}\right)$ & $Q_{\ell e q u}^{(1)}$ & $\left(\bar{\ell}_{i}^{a} e_{j}\right) \varepsilon_{a b}\left(\bar{q}_{k}^{b} u_{l}\right)$ \\
& & & $Q_{\ell e q u}^{(3)}$ & $\left(\bar{\ell}_{i}^{a} \sigma_{\mu \nu} e_{a}\right) \varepsilon_{a b}\left(\bar{q}_{k}^{b} \sigma^{\mu \nu} u_{l}\right)$ \\
\hline
\end{tabular}

Table 5. Complete list of the dimension-6 operators (invariant under the SM gauge group) which contribute to the LFV observables under consideration at the tree or at the one-loop level.

following we will only consider one of these combinations which avoids the introduction of combinatorial factors. This can be achieved by the requirement $i \geq k, j \geq l$ for $Q_{\ell \ell, e e}^{i j k l}$, so that the relevant part of the Lagrangian can be written as:

$$
\mathcal{L}=\frac{1}{\Lambda^{2}} \sum_{i j k l, i \geq k, j \geq l}\left(C_{\ell \ell}^{i j k l} Q_{\ell \ell}^{i j k l}+C_{e e}^{i j k l} Q_{e e}^{i j k l}\right)+\frac{1}{\Lambda^{2}} \sum_{i j k l} C_{\ell e}^{i j k l} Q_{\ell e}^{i j k l} .
$$

Note that for $C_{\ell e}^{i j k l}$ all possible flavor index permutations correspond to different operators. Due to the hermiticity of the Lagrangian we find the additional relations like $C_{\ell \ell}^{i j k l}=C_{\ell \ell}^{j i l k \star}$. Similar ones hold for all four-fermion operators.

The dominant contributions to the processes considered in this article are given by diagrams with flavor changing gauge boson vertices or contact 4 -fermion vertices. However, to preserve gauge-invariance, also Goldstone boson exchanges has to be taken into account even if, with few exceptions of mixed $W^{ \pm} G^{\mp}$ diagrams, they are suppressed by additional powers of light lepton masses over $v$, the Higgs field VEV. In general, the operators listed in table 5 give rise also to flavor violating physical Higgs boson couplings. We neglect them in our analysis as they are again of the higher order in $m_{\ell} / m_{h^{0}}$.

The $\left(\varphi^{\dagger} \varphi\right)\left(\bar{\ell}_{i} e_{j} \varphi\right)$ operator does not contain gauge boson fields and modifies only Higgs and Goldstone boson couplings, which in principle could affect our results. However, it gives also new $\mathcal{O}\left(1 / \Lambda^{2}\right)$ contribution to the charged lepton mass matrix:

$$
m_{f i}^{\ell}=\frac{v}{\sqrt{2}} Y_{f}^{\ell} \delta_{f i}+\frac{v^{3}}{2 \sqrt{2} \Lambda^{2}} C_{e \varphi 3}^{f i} .
$$

The necessary rediagonalization of lepton masses has the effect of modifying the relation between the Yukawa coupling and the charged lepton masses (and the PMNS matrix). 
However, one can see that in the triple Goldstone boson couplings to leptons still the physical lepton masses and the physical PMNS matrix enter so the $Q_{e \phi 3}^{f i}$ does not generate flavor violation in these couplings. The triple coupling of the physical Higgs boson $h^{0}$ to charged leptons, as well as all quadruple and quintuple vertices derived from $Q_{e \phi 3}^{f i}$ can still be flavor violating. Nonetheless, their contributions to the processes discussed below vanish or are small due to an additional suppression of $m_{\ell} / m_{h^{0}}$, compared to the dominant contributions from $Q_{\varphi e}, Q_{\varphi \ell}^{(1)}$ and $Q_{\varphi \ell}^{(3)}$ operators. ${ }^{2}$ Thus, we neglect this operator (and thus the entire $\ell \ell \varphi^{3}$ class) in our analysis, provided that the rediagonalization of the lepton mass matrix has been performed.

The operators of the $\ell \ell X \varphi$ class (as defined in table 5) can give rise to both radiative lepton decays and to three-body neutral current lepton decays already at the tree-level. The 4-lepton $\ell \ell \ell \ell$ operators and the operators of the $\ell \ell \varphi^{2} D$ class can contribute to $\ell_{i} \rightarrow \ell_{j} \ell_{k} \ell_{l}$ decays at the tree-level and to $\ell_{i} \rightarrow \ell_{f} \gamma$ decays at the 1-loop level. Finally, the operators of the $\ell \ell q q$ class can contribute to both types of decays only at the 1-loop level. However, for 3-body decays we are only interested in the tree-level contributions and concerning the radiative lepton decays, it turns out that only $Q_{\text {lequ }}^{(3)}$ gives a non-zero contribution.

In the appendix we list the Feynman rules arising from the operators given in table 5 which are necessary in order to calculate the flavor observables discussed in the next sections.

\section{Observables related to the effective lepton-photon coupling}

As outlined in the introduction, observables related to effective lepton-photon coupling: radiative lepton decays (especially $\mu \rightarrow e \gamma$ ), EDMs of charged leptons and their anomalous magnetic moments are very sensitive to NP and allow to constrain stringently the relevant Wilson coefficients.

The general form of the flavor violating photon-lepton vertex can be written as:

$$
V_{\ell \ell \gamma}^{f i \mu}=\frac{i}{\Lambda^{2}}\left[\gamma^{\mu}\left(F_{V L}^{f i} P_{L}+F_{V R}^{f i} P_{R}\right)+\left(F_{S L}^{f i} P_{L}+F_{S R}^{f i} P_{R}\right) q^{\mu}+\left(F_{T L}^{f i} i \sigma^{\mu \nu} P_{L}+F_{T R}^{f i} i \sigma^{\mu \nu} P_{R}\right) q_{\nu}\right] .
$$

In this section we calculate the expressions for the formfactors in eq. (3.1) necessary to calculate the branching ratio for the $\ell_{i} \rightarrow \ell_{f} \gamma$ decays (with $i>f$ ) at the 1-loop level up the order $1 / \Lambda^{2}$. In addition, the obtained results are directly related to the anomalous magnetic moments and the electric dipole moments (EDM) of leptons after setting $f=i$.

\subsection{Radiative lepton decays}

Gauge-invariance requires that $F_{V L}$ and $F_{V R}$ must vanish for on-shell external particles. The form-factors $F_{S L}$ and $F_{S R}$ do not contribute to the $\ell_{i} \rightarrow \ell_{f} \gamma$ decay amplitude and the branching ratio can be expressed in terms of $F_{T L}^{f i}$ and $F_{T R}^{f i}$ only:

$$
\operatorname{Br}\left[\ell_{i} \rightarrow \ell_{f} \gamma\right]=\frac{m_{\ell_{i}}^{3}}{16 \pi \Lambda^{4} \Gamma_{\ell_{i}}}\left(\left|F_{T R}^{f i}\right|^{2}+\left|F_{T L}^{f i}\right|^{2}\right)
$$

\footnotetext{
${ }^{2} O_{e \varphi 3}^{f i}$ generates flavour-changing couplings of the SM-Higgs. The resulting effects have been studied in refs. [46-48].
} 


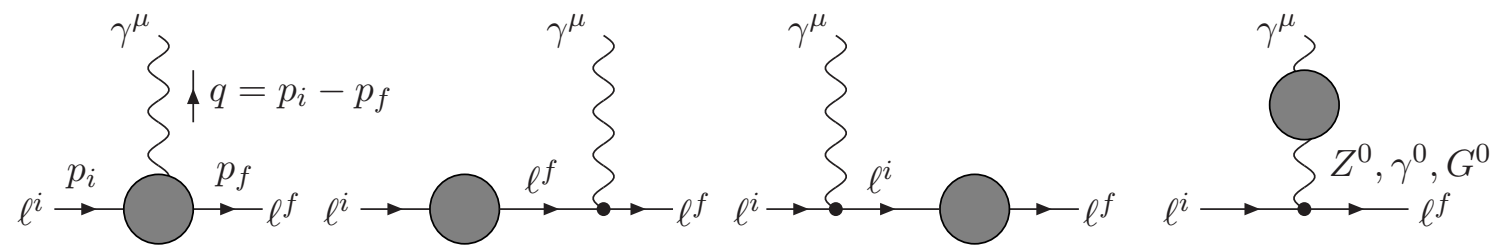

Figure 1. Topologies of diagrams contributing to radiative decay $\ell^{i} \rightarrow \ell^{f} \gamma$.

The total decay width of the muon is given by $\Gamma_{\mu}=\frac{G_{F}^{2} m_{\mu}^{5}}{192 \pi^{3}}$ and for the tau lepton $\Gamma_{\tau}$ includes the leptonic and hadronic decay channels.

Only the operators $Q_{e W}$ (here $W$ denotes the neutral gauge boson of the $\mathrm{SU}(2)_{L}$ gauge group) and $Q_{e B}$ can contribute to $F_{T L, R}^{f i}$ at the tree-level. If their coefficients are comparable to other Wilson coefficient of the dimension 6 operators, they dominate the effective photon-lepton vertex, with the form-factors simply given by $\left(v=\frac{2 M_{W}}{g_{2}}\right)$ :

$$
F_{T R}^{f i}=F_{T L}^{i f \star}=v \sqrt{2}\left(c_{W} C_{e B}^{f i}-s_{W} C_{e W}^{f i}\right) \equiv v \sqrt{2} C_{\gamma}^{f i} .
$$

However, in a renormalizable theory of NP the operators $Q_{e W}$ and $Q_{e B}$ can only be generated at the loop-level while other operators, like the effective four-lepton couplings, can already be generated at the tree-level. In some extensions of the SM $C_{e W}$ and $C_{e B}$ may even not be generated at all [49]. Thus, comparable (or even dominant) contributions to the flavor violating lepton-lepton-photon vertex can come from other dimension-6 operators, which for consistency should be included at the 1-loop level. The generic topologies of the diagrams which could contribute to $\ell_{i} \rightarrow \ell_{f} \gamma$ at the 1-loop level in the order $1 / \Lambda^{2}$ and the relevant momenta assignments are shown in figure 1.

The list of all 1-loop diagrams contributing to the effective lepton-photon vertex is given in figure 2 (lepton self-energy contributions) and figure 3 (1-particle irreducible vertex corrections). The diagrams contributing to photon-photon and $Z^{0}$-photon self-energies are the same as in the SM (with $W$ bosons, charged ghosts, charged Goldstone bosons and charged fermion as virtual particles). In our loop calculations we do not take into account flavor violating photon and $Z^{0}$ couplings generated at the one-loop level by the operators $Q_{e W}$ and $Q_{e B}$ because if their coefficients are non-negligible, than already the tree-level contribution of eq. (3.3) would dominate the whole process anyway.

Our final 1-loop results for the form-factors $F_{T L}$ and $F_{T R}$ are given in table 6 . We group them into subsets; within these subsets the vector form-factors $F_{V L}$ and $F_{V R}$ vanish separately in the on-shell limit. We kept only the leading term in $1 / \Lambda^{2}$ and we expand all diagrams involving $Z^{0}$ and $W$ bosons (or the associated Goldstone bosons) in the charged lepton masses, keeping only the leading terms in $m_{\ell} / m_{W}, m_{\ell} / m_{Z}$. For this expansion we used two independent approaches for calculating the diagrams. In the first approach the exact calculation of all loop integrals is performed, followed by their expansion in the external momenta. In the second approach we used asymptotic expansion [50] and expanded the diagrams in external momenta before performing the loop integrals, finding the same result as with the first approach. The final expressions collected in table 6 are compact and simple. 


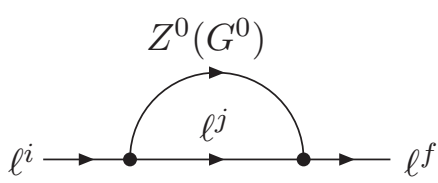

a)

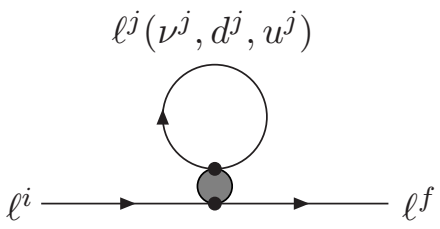

d)

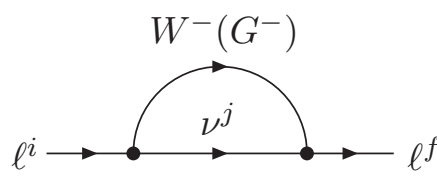

b)

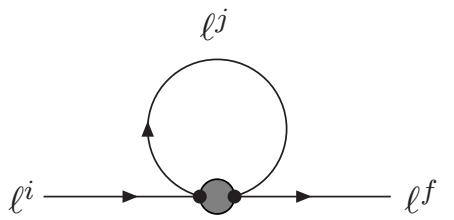

e)

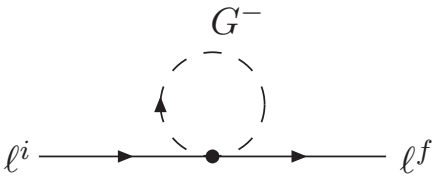

c)

Figure 2. Diagrams contributing to LFV self-energy of charged leptons.

As mentioned above, if the external particles in the flavor violating lepton-photon vertex are on-shell, gauge invariance requires $F_{V L}^{f i}=F_{V R}^{f i}=0$ for $i \neq f$. As the diagrams involving dimension- 6 vertices may have complicated tensor structures, the vanishing of the $F_{V L}$ and $F_{V R}$ is an important check of our calculation. As an additional check we performed the whole calculation in a general $R_{\xi}$ gauge finding that the $\xi$ dependence cancels for all form-factors. Here one should keep in mind that taking into account only 1PI irreducible diagrams is sufficient for the calculation of $F_{T L}, F_{T R}$ - however, taking into account also lepton, photon and mixing photon- $Z^{0}$ self-energies diagrams is obligatory to cancel completely the vector form-factors and to get a gauge-independent renormalization constant for the electric charge.

We see that in the final result, at the 1-loop level and in the first order of expansions in $1 / \Lambda^{2}$ and $m_{l}$, only the five Wilson coefficients $C_{\ell e}^{f j j i}, C_{\ell e q u}^{(3) f i j j}, C_{\varphi \ell}^{(3) f i}, C_{\varphi e}^{f i}$ and $C_{\varphi \ell}^{(1) f i}$ enter, while the contribution of all other Wilson coefficients is zero.

It is interesting to note that the term proportional to $C_{\text {lequ }}^{(3)}$ is the only one containing a divergence. This divergence must be canceled by a counter-term to $Q_{e W}$ and/or $Q_{e B}$. The appearance of this divergence can be understood by looking at a UV complete theory of NP. Consider as an example a theory with a heavy scalar particle. Directly calculating the contributions to $F_{T L}$ and $F_{T R}$ in the full theory one would obtain a finite result. However, when matching to full theory on the SM extended with dimension-6 operators the situation is more complicated: integrating out the heavy particle at the matching scale $\Lambda$ gives rise to $C_{e W}$ and $C_{e B}$ at the loop-level and $C_{\text {lequ }}^{(3)}$ at the tree-level. However, as all Wilson coefficients, $C_{e W}$ and $C_{e B}$ can only contain the hard part of the corresponding loop-contribution while the soft part must be canceled by the loop-contribution of $Q_{\text {lequ }}^{(3)}$ to $C_{e W}$ and $C_{e B}$ in an effective theory. It turns out that the hard part which contributes to $C_{e W}$ and $C_{e B}$ has a infrared divergence which is canceled by the UV divergence of the soft part (as can be best seen using asymptotic expansion). Comparing this result with the one in the full theory we see that the $\mu$-dependence in the contribution of $C_{\text {lequ }}^{(3)}$ to $F_{T L}$ and 


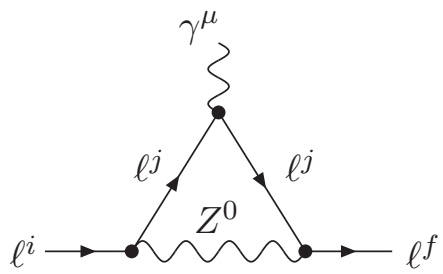

a)

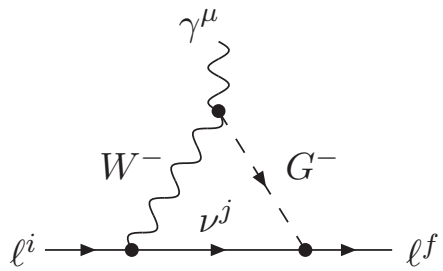

d)

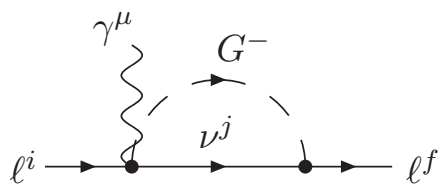

g)

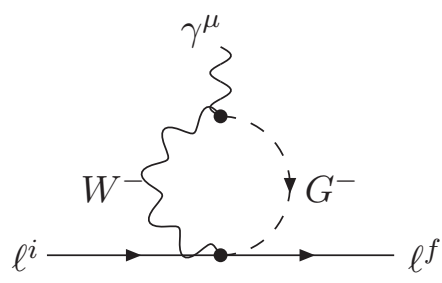

j)

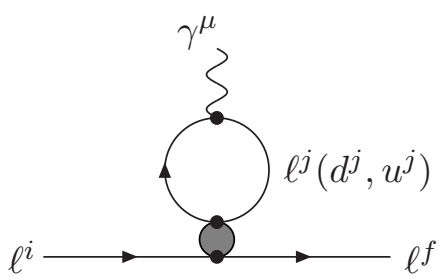

m)

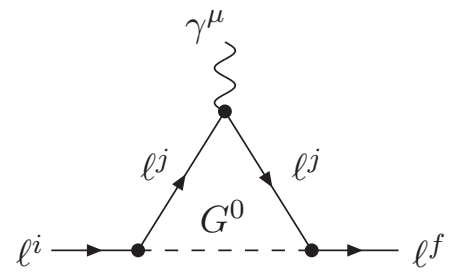

b)

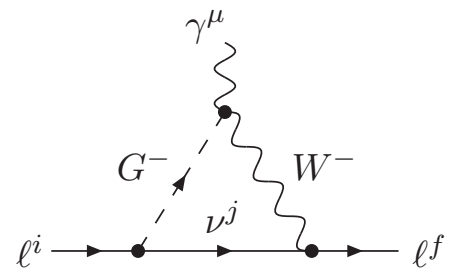

e)

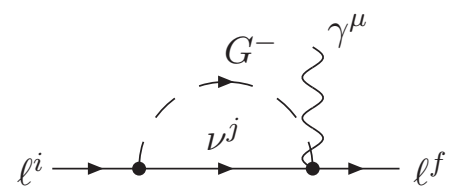

h)

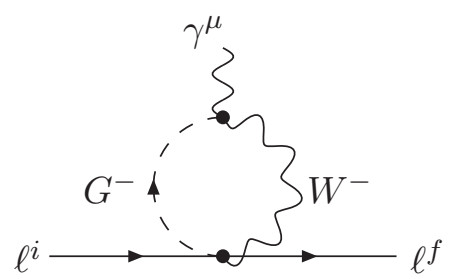

k)

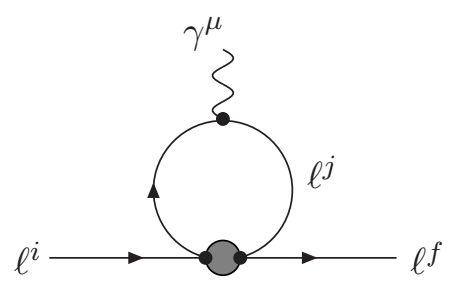

n)

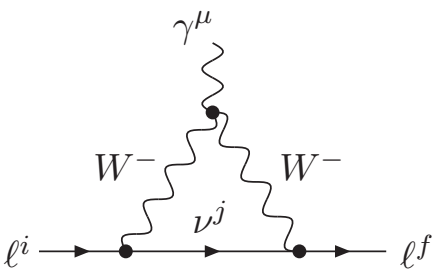

c)

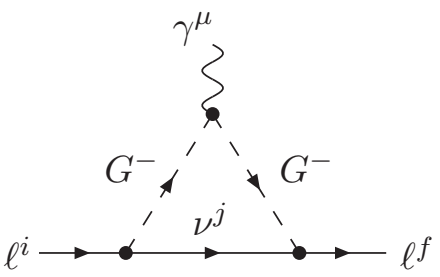

f)

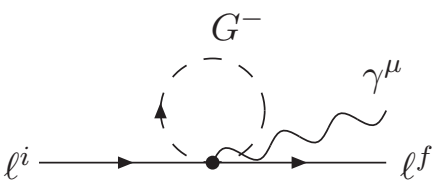

i)

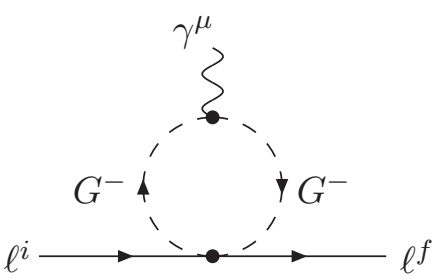

1)

Figure 3. 3-point 1PI diagrams contributing to the radiative charged lepton decay $\ell^{i} \rightarrow \ell^{f} \gamma$ at the 1-loop level.

$F_{T R}$ must be replaced by the mass of the heavy scalar, i.e. $\Lambda$. In our numerical analysis we neglect (possible but rather exotic in the lepton sector) contributions from $Q_{\text {lequ }}^{(3)}$ operator - coefficients of such lepton-quark contact terms can be independently constrained using the LHC measurements [51]. 


\begin{tabular}{|c|c|}
\hline Group (diagrams of figures 2,3 ) & Tensor form-factors \\
\hline \multirow{3}{*}{$Z^{0}\left(3 \mathrm{a}, 2 \mathrm{a}\left(Z^{0}\right)\right)$} & 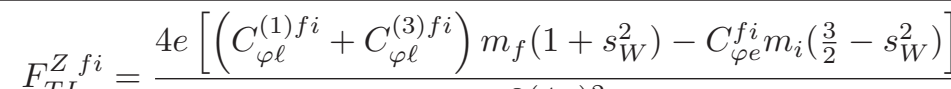 \\
\hline & $F^{Z f i}=\frac{4 e\left[\left(C_{\varphi \ell}^{(1) f i}+C_{\varphi l}^{(3) f i}\right) m_{i}\left(1+s_{W}^{2}\right)-C_{\varphi e}^{f i} m_{f}\left(\frac{3}{2}-s_{W}^{2}\right)\right]}{2(4 \pi)^{2}}$ \\
\hline & $F_{T R}=-3(4 \pi)^{2}$ \\
\hline \multirow[t]{2}{*}{$G^{0}\left(3 \mathrm{~b}, 2 \mathrm{a}\left(G^{0}\right)\right)$} & \multirow{2}{*}{$\begin{array}{l}F_{T L}^{G^{0} f i}=0 \\
F_{T R}^{G^{0} f i}=0\end{array}$} \\
\hline & \\
\hline \multirow{4}{*}{$W(3 \mathrm{c}, \mathrm{d}, \mathrm{e}, \mathrm{j}, \mathrm{k}, 2 \mathrm{~b}(W))$} & \multirow{4}{*}{$\begin{aligned} F_{T L}^{W f i} & =-\frac{10 e m_{f} C_{\varphi \ell}^{(3) f i}}{3(4 \pi)^{2}} \\
F_{T R}^{W f i} & =-\frac{10 e m_{i} C_{\varphi \ell}^{(3) f i}}{3(4 \pi)^{2}}\end{aligned}$} \\
\hline & \\
\hline & \\
\hline & \\
\hline \multirow[t]{2}{*}{$G^{ \pm}\left(3 \mathrm{f}, \mathrm{g}, \mathrm{h}, 2 \mathrm{~b}\left(G^{ \pm}\right)\right)$} & $F_{T L}^{G^{ \pm}} f i=0$ \\
\hline & $F_{T R}^{G^{ \pm}} f i=0$ \\
\hline \multirow[t]{2}{*}{$W G$ "bubble" $(3 \mathrm{i}, 1,2 \mathrm{c})$} & $F_{T L}^{W G f i}=0$ \\
\hline & $F_{T R}^{W G f i}=0$ \\
\hline \multirow[t]{2}{*}{ contact 4 -fermion $(3 \mathrm{~m}, 2 \mathrm{~d})$} & $F_{T L}^{4 f f i}=-\frac{16 e}{3(4 \pi)^{2}} \sum_{j=1}^{3} C_{\ell e q u}^{(3) f i j j \star} m_{u_{j}}\left(\Delta-\log \frac{m_{u_{j}}^{2}}{\mu^{2}}\right)$ \\
\hline & $F_{T R}^{4 f f i}=-\frac{16 e}{3(4 \pi)^{2}} \sum_{j=1}^{3} C_{\ell e q u}^{(3) f i j j} m_{u_{j}}\left(\Delta-\log \frac{m_{u_{j}}^{2}}{\mu^{2}}\right)$ \\
\hline contact 4-lepton (3n, 2e) & $\begin{aligned} F_{T L}^{4 \ell f i} & =\frac{2 e}{(4 \pi)^{2}} \sum_{j=1}^{3} C_{\ell e}^{f j j i} m_{j} \\
F_{T R}^{4 \ell f i} & =\frac{2 e}{(4 \pi)^{2}} \sum_{j=1}^{3} C_{\ell e}^{j i f j} m_{j}\end{aligned}$ \\
\hline
\end{tabular}

Table 6. One-loop contributions to form factors $F_{T L}^{f i}$ and $F_{T L}^{f i}$ giving rise to $\ell_{i} \rightarrow \ell_{f} \gamma$ up to order $1 / \Lambda^{2}$.

\subsection{Anomalous magnetic moments and electric dipole moments}

The form-factors listed in table 6 for $f=i$ can directly be used to calculate also the electric dipole moments of charged leptons and the contribution (in addition to the SM) to their anomalous magnetic moments:

$$
\begin{aligned}
d_{\ell_{i}} & =\frac{-1}{\Lambda^{2}} \operatorname{Im}\left[F_{T R}^{i i}\right], \\
a_{\ell_{i}} & =\frac{2 m_{\ell_{i}}}{e \Lambda^{2}} \operatorname{Re}\left[F_{T R}^{i i}\right] .
\end{aligned}
$$

The experimental bounds on the EDM of charged leptons are given in table 3.

The anomalous magnetic moment of the electron is usually used to determine the fine structure constant, but determining $\alpha_{e m}$ from rubidium atom experiments [52], one can still use it for obtaining bounds on NP [17, 53, 54]. For the anomalous magnetic moment of 
the muon there is the long known discrepancy between experiment and the SM prediction for $a_{\mu}=(g-2) / 2[55-59]$ :

$$
\Delta a_{\mu}=a_{\mu}^{e x p}-a_{\mu}^{S M} \approx(2.7 \pm 0.8) \times 10^{-9} .
$$

This discrepancy could point towards physics beyond the SM and, if verified, could make the search for $\ell_{i} \rightarrow \ell_{f} \gamma$ decay even more promising, as both processes depend on the operators with formally the same field and Dirac structure, differing only by the choice of flavor indices.

The current experimental limit on the anomalous magnetic moment of the tau lepton is rather weak, but it can be improved in the future [60]:

$$
-0.052 \leq a_{\tau} \leq 0.013
$$

\section{$4 \quad \ell_{i} \rightarrow \ell_{j} \ell_{k} \bar{\ell}_{l}$ decay rate}

LFV operators of dimension- 6 also give contributions to another set of experimentally strongly constrained decays, namely decays of heavy charged lepton into three lighter charged leptons. ${ }^{3}$ Such decays can be generated already at the tree-level by $Z^{0}$ and neutral Goldstone boson exchange, flavor violating photon couplings generated by $Q_{e W}$ and $Q_{e B}$ operators, or even directly by the 4 -lepton operators. In this section we list the general expressions for the lowest order contributions to all such 3-body charged lepton decays. Since all operators enter already at the tree-level we choose not to consider loop-diagrams for these processes.

We split the expressions for the $\ell_{i} \rightarrow \ell_{j} \ell_{k} \bar{\ell}_{l}$ decays into 3 groups, depending on composition of the final state leptons:

(A) Three leptons of the same flavor: $\mu^{ \pm} \rightarrow e^{ \pm} e^{+} e^{-}, \tau^{ \pm} \rightarrow e^{ \pm} e^{+} e^{-}$and $\tau^{ \pm} \rightarrow \mu^{ \pm} \mu^{+} \mu^{-}$.

(B) Three distinguishable leptons: $\tau^{ \pm} \rightarrow e^{ \pm} \mu^{+} \mu^{-}$and $\tau^{ \pm} \rightarrow \mu^{ \pm} e^{+} e^{-}$.

(C) Two lepton of the same flavor and charge and one with different flavor and opposite charge: $\tau^{ \pm} \rightarrow e^{\mp} \mu^{ \pm} \mu^{ \pm}$and $\tau^{ \pm} \rightarrow \mu^{\mp} e^{ \pm} e^{ \pm}$.

We decompose the amplitude $A$ for the decay $\ell_{i} \rightarrow \ell_{j} \ell_{k} \bar{\ell}_{l}$ as

$$
A=A_{0}+A_{\gamma},
$$

where $A_{0}$ contains all operators for which one can neglect the momenta of the external leptons and $A_{\gamma}$ is the photon contribution generated in our approximation by $Q_{e W}$ and $Q_{e B}$ only. The amplitude $A_{0}$ can without loss of generality be written as: ${ }^{4}$

$$
A_{0}=\frac{1}{\Lambda^{2}} \sum_{I} C_{I}\left[\bar{u}\left(p_{j}\right) Q_{I} u\left(p_{i}\right)\right]\left[\bar{u}\left(p_{k}\right) Q_{I}^{\prime} v\left(p_{l}\right)\right]
$$

\footnotetext{
${ }^{3}$ Experimental bounds are usually given on positively charged muon decays, as they do not form bound state with atoms what would decrease the accuracy of measurements [61].

${ }^{4}$ We define the amplitude in such a way that calculating a diagram equals $i A$, which means that the Wilson coefficients are purely real in the absence of $\mathrm{CP}$ violation.
} 


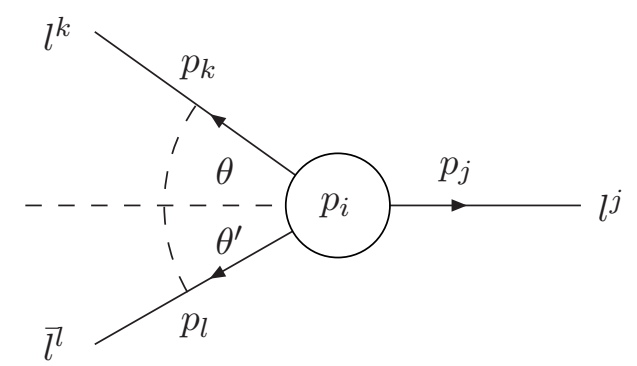

Figure 4. Kinematics of $\ell_{i} \rightarrow \ell_{j} \ell_{k} \bar{\ell}_{l}$ decay in the CMS frame.

with the momenta assignments shown in figure 4 . The basis of quadrilinears $Q_{I} \times Q_{I}^{\prime}$ is given by:

$$
\begin{aligned}
O_{V X Y} & =\gamma^{\mu} P_{X} \times \gamma^{\mu} P_{Y}, \\
O_{S X Y} & =P_{X} \times P_{Y}, \\
O_{T X} & =\sigma^{\mu \nu} \times \sigma^{\mu \nu} P_{X},
\end{aligned}
$$

where $X, Y$ stands for the chiralities $L$ and $R$. For processes with two identical leptons in the final state one needs to include crossed diagrams in which the different spinor ordering $\left[\bar{u}\left(p_{j}\right) Q_{I} u\left(p_{l}\right)\right]\left[\bar{u}\left(p_{k}\right) Q_{I}^{\prime} v\left(p_{i}\right)\right]$ appears. However, one can always reduced these contributions to form given in eq. (4.2) by the appropriate Fierz transformations (see e.g. [62]).

The contributions from photon exchange for various types of decays (A), (B), (C) read (retaining only $1 / \Lambda^{2}$ terms):

$$
\begin{aligned}
& A_{\gamma}^{(A)}=\frac{e v}{\Lambda^{2}}\left(\frac{1}{\left(p_{i}-p_{j}\right)^{2}}\left[\bar{u}\left(p_{j}\right) i \sigma^{\mu \nu}\left(C_{\gamma L} P_{L}+C_{\gamma R} P_{R}\right)\left(p_{i}-p_{j}\right)_{\nu} u\left(p_{i}\right)\right]\left[\bar{u}\left(p_{k}\right) \gamma_{\mu} v\left(p_{l}\right)\right]-\left(p_{j} \leftrightarrow p_{k}\right)\right) \\
& A_{\gamma}^{(B)}=\frac{e v}{\Lambda^{2}} \frac{1}{\left(p_{i}-p_{j}\right)^{2}}\left[\bar{u}\left(p_{j}\right) i \sigma^{\mu \nu}\left(C_{\gamma L} P_{L}+C_{\gamma R} P_{R}\right)\left(p_{i}-p_{j}\right)_{\nu} u\left(p_{i}\right)\right]\left[\bar{u}\left(p_{k}\right) \gamma_{\mu} v\left(p_{l}\right)\right] \\
& A_{\gamma}^{(C)}=0
\end{aligned}
$$

In eq. (4.2) and eq. (4.4) we did not write explicitly flavor indices of $C_{I}, C_{\gamma}$ but we specify them later in the next section.

The general expression for the spin averaged square matrix element $\mathcal{M}=\frac{1}{2} \sum_{\text {pol }}|A|^{2}$ is complicated, but due to the hierarchy of the charged lepton masses, in most cases it is sufficient to assume $m_{i} \equiv M \gg m_{j}, m_{k}, m_{l}$ and neglect the lighter lepton masses (which also eliminates the contribution of Goldstone bosons). Only the contribution from the photon penguin requires more care due to singularity of photon propagator for small momenta. For the photon penguin, in order to get the correct final result one needs to expand matrix element and phase space kinematics at least up to the order of $m^{2} / M^{2}$. Then, using standard expressions for 3-particle phase space one can integrate the matrix element and 
obtain the branching ratios (for comparison see [26]):

$$
\begin{aligned}
\operatorname{Br}\left(\ell_{i} \rightarrow \ell_{j} \ell_{k} \bar{\ell}_{l}\right)= & \frac{N_{c} M^{5}}{6144 \pi^{3} \Lambda^{4} \Gamma_{\ell_{i}}}\left(4\left(\left|C_{V L L}\right|^{2}+\left|C_{V R R}\right|^{2}+\left|C_{V L R}\right|^{2}+\left|C_{V R L}\right|^{2}\right)\right. \\
& +\left|C_{S L L}\right|^{2}+\left|C_{S R R}\right|^{2}+\left|C_{S L R}\right|^{2}+\left|C_{S R L}\right|^{2} \\
& \left.+48\left(\left|C_{T L}\right|^{2}+\left|C_{T R}\right|^{2}\right)+X_{\gamma}\right)
\end{aligned}
$$

where $N_{c}=1 / 2$ if two of the final state leptons are identical, $N_{c}=1$ in all other cases and $\Gamma_{\ell_{i}}$ is the total decay width of the initial lepton. The photon penguin contribution reads:

$$
\begin{aligned}
X_{\gamma}^{(A)}= & -\frac{16 e v}{M} \operatorname{Re}\left[\left(2 C_{V L L}+C_{V L R}-\frac{1}{2} C_{S L R}\right) C_{\gamma R}^{\star}+\left(2 C_{V R R}+C_{V R L}-\frac{1}{2} C_{S R L}\right) C_{\gamma L}^{\star}\right] \\
& +\frac{64 e^{2} v^{2}}{M^{2}}\left(\log \frac{M^{2}}{m^{2}}-\frac{11}{4}\right)\left(\left|C_{\gamma L}\right|^{2}+\left|C_{\gamma R}\right|^{2}\right) \\
X_{\gamma}^{(B)}= & -\frac{16 e v}{M} \operatorname{Re}\left[\left(C_{V L L}+C_{V L R}\right) C_{\gamma R}^{\star}+\left(C_{V R R}+C_{V R L}\right) C_{\gamma L}^{\star}\right] \\
& +\frac{32 e^{2} v^{2}}{M^{2}}\left(\log \frac{M^{2}}{m^{2}}-3\right)\left(\left|C_{\gamma L}\right|^{2}+\left|C_{\gamma R}\right|^{2}\right) \\
X_{\gamma}^{(C)}= & 0
\end{aligned}
$$

\subsection{Decay $\ell_{i} \rightarrow \ell_{j} \ell_{j} \bar{\ell}_{j}$}

This option responds to the physical decays $\mu \rightarrow 3 e, \tau \rightarrow 3 e$ and $\tau \rightarrow 3 \mu$. In general, at the tree-level diagrams mediated by photon, $Z^{0}$, the neutral Goldstone boson and 4-lepton contact terms can contribute to the matrix element. The quantities $C_{X}$ in eq. (4.5) can be expressed in terms of Wilson coefficients of operators in table 5 as (with $C_{\gamma}^{j i}$ defined in eq. (3.3)):

$$
\begin{aligned}
C_{V L L} & =2\left(\left(2 s_{W}^{2}-1\right)\left(C_{\varphi \ell}^{(1) j i}+C_{\varphi \ell}^{(3) j i}\right)+C_{\ell \ell}^{j i j j}\right) \\
C_{V R R} & =2\left(2 s_{W}^{2} C_{\varphi e}^{j i}+C_{e e}^{j i j j}\right) \\
C_{V L R} & =-\frac{1}{2} C_{S R L}=2 s_{W}^{2}\left(C_{\varphi \ell}^{(1) j i}+C_{\varphi \ell}^{(3) j i}\right)+C_{\ell e}^{j i j j} \\
C_{V R L} & =-\frac{1}{2} C_{S L R}=\left(2 s_{W}^{2}-1\right) C_{\varphi e}^{j i}+C_{\ell e}^{j j j i} \\
C_{S L L} & =C_{S R R}=C_{T L}=C_{T R}=0 \\
C_{\gamma L} & =\sqrt{2} C_{\gamma}^{i j \star} \\
C_{\gamma R} & =\sqrt{2} C_{\gamma}^{j i}
\end{aligned}
$$




\subsection{Decay $\ell_{i} \rightarrow \ell_{j} \ell_{k} \bar{\ell}_{k}$}

Such a decay can be realized as $\tau^{ \pm} \rightarrow e^{ \pm} \mu^{+} \mu^{-} e$ or $\tau^{ \pm} \rightarrow \mu^{ \pm} e^{+} e^{-}$. The coefficients $C_{X}$ read:

$$
\begin{aligned}
C_{V L L} & =\left(2 s_{W}^{2}-1\right)\left(C_{\varphi \ell}^{(1) j i}+C_{\varphi l}^{(3) j i}\right)+C_{\ell \ell}^{j i k k} \\
C_{V R R} & =2 s_{W}^{2} C_{\varphi e}^{j i}+C_{e e}^{j i k k} \\
C_{V L R} & =2 s_{W}^{2}\left(C_{\varphi \ell}^{(1) j i}+C_{\varphi \ell}^{(3) j i}\right)+C_{\ell e}^{j i k k} \\
C_{V R L} & =\left(2 s_{W}^{2}-1\right) C_{\varphi e}^{j i}+C_{\ell e}^{j k k i} \\
C_{S L R} & =-2 C_{\ell e}^{j k k i} \\
C_{S R L} & =-2 C_{\ell e}^{j i k k} \\
C_{S L L} & =C_{S R R}=C_{T L}=C_{T R}=0 \\
C_{\gamma L} & =\sqrt{2} C_{\gamma}^{i j \star} \\
C_{\gamma R} & =\sqrt{2} C_{\gamma}^{j i}
\end{aligned}
$$

\subsection{Decay $\ell_{i}^{ \pm} \rightarrow \bar{\ell}_{j}^{\mp} \ell_{k}^{ \pm} \ell_{k}^{ \pm}$}

Again, only $\tau$ lepton can decay into such channels, $\tau^{ \pm} \rightarrow e^{\mp} \mu^{ \pm} \mu^{ \pm}$or $\tau^{ \pm} \rightarrow \mu^{\mp} e^{\mp} e^{\mp}$. In this case photon and $Z^{0}$-mediated diagrams are suppressed by $1 / \Lambda^{4}$ and only contact 4 -lepton diagram can contribute to these (rather exotic) process. The coefficients $C_{X}$ are given by:

$$
\begin{aligned}
C_{V L L} & =2 C_{\ell \ell}^{k i k j} \\
C_{V R R} & =2 C_{e e}^{k i k j} \\
C_{V L R} & =-\frac{1}{2} C_{S R L}=C_{\ell e}^{k i k j} \\
C_{V R L} & =-\frac{1}{2} C_{S L R}=C_{\ell e}^{k j k i} \\
C_{S L L} & =C_{S R R}=C_{T L}=C_{T R}=0 \\
C_{\gamma L} & =C_{\gamma R}=0
\end{aligned}
$$

\section{Lepton flavor violating $Z^{0}$ decays}

The branching ratio for the lepton flavor violating decays of a $Z^{0}$ boson $Z^{0} \rightarrow \ell_{f}^{-} \ell_{i}^{+}$is given by:

$$
\operatorname{Br}\left[Z^{0} \rightarrow \ell_{f}^{ \pm} \ell_{i}^{\mp}\right]=\frac{m_{Z}}{24 \pi \Gamma_{Z}}\left[\frac{m_{Z}^{2}}{2}\left(\left|C_{f i}^{Z R}\right|^{2}+\left|C_{f i}^{Z L}\right|^{2}\right)+\left|\Gamma_{f i}^{Z L}\right|^{2}+\left|\Gamma_{f i}^{Z R}\right|^{2}\right],
$$

where $\Gamma_{Z} \approx 2.495 \mathrm{GeV}$ is the total decay width of the $Z^{0}$ boson. We included all tree-level contributions and

$$
\begin{aligned}
\Gamma_{f i}^{Z L} & =\frac{e}{2 s_{W} c_{W}}\left(\frac{v^{2}}{\Lambda^{2}}\left(C_{\varphi l}^{(1) f i}+C_{\varphi l}^{(3) f i}\right)+\left(1-2 s_{W}^{2}\right) \delta_{f i}\right), \\
\Gamma_{f i}^{Z R} & =\frac{e}{2 s_{W} c_{W}}\left(\frac{v^{2}}{\Lambda^{2}} C_{\varphi e}^{f i}-2 s_{W}^{2} \delta_{f i}\right), \\
C_{f i}^{Z R} & =C_{i f}^{Z L \star}=-\frac{v}{\sqrt{2} \Lambda^{2}} C_{Z}^{f i}
\end{aligned}
$$




\begin{tabular}{|c|c|}
\hline Process & Experimental bound \\
\hline \hline $\operatorname{Br}\left[Z^{0} \rightarrow \mu^{ \pm} e^{\mp}\right]$ & $1.7 \times 10^{-6}[64]$ \\
\hline $\operatorname{Br}\left[Z^{0} \rightarrow \tau^{ \pm} e^{\mp}\right]$ & $9.8 \times 10^{-6}[64]$ \\
\hline $\operatorname{Br}\left[Z^{0} \rightarrow \tau^{ \pm} \mu^{\mp}\right]$ & $1.2 \times 10^{-5}[64]$ \\
\hline
\end{tabular}

Table 7. Experimental upper limits (95\% CL) on the lepton flavor violating $Z^{0}$ decay rates.

where $C_{Z}^{f i}$ is defined as

$$
C_{Z}^{f i}=\left(s_{W} C_{e B}^{f i}+c_{W} C_{e W}^{f i}\right)
$$

The experimental bounds on these decays are given in table 7 . Their current sensitivities are not as good as for the other lepton flavor violating decays but a future linear collider could significantly improve them [63]. Note that theoretical prediction in eq. (5.1) is for the decay $Z^{0} \rightarrow \ell_{f}^{-} \ell_{i}^{+}$or $Z^{0} \rightarrow \ell_{f}^{+} \ell_{i}^{-}$while the experimental values are for the $\operatorname{sum} Z^{0} \rightarrow \ell_{f}^{-} \ell_{i}^{+}+\ell_{i}^{-} \ell_{f}^{+}$. Therefore, eq. (5.1) must be multiplied by a factor of 2 in order to compare it to the experimental values.

\section{$6 \quad$ Numerical analysis}

In the absence of fine-tuning and accidental cancellations the Wilson coefficients of the flavor changing 4-lepton operators and of the flavor changing $Z^{0}$-lepton-lepton vertex are most stringently constrained by the three-body charged lepton decays, while $C_{\gamma}^{f i}=c_{W} C_{e B}^{f i}-s_{W} C_{e W}^{f i}$ is best restricted by the radiative lepton decays. Henceforth, as a first approximation one can obtain the approximate bounds on $C_{\gamma}^{f i}$ from the experimental upper limits on $\operatorname{Br}\left[\ell_{i} \rightarrow \ell_{f} \gamma\right]$, assuming that all other Wilson coefficients are negligible:

$$
\begin{aligned}
& \sqrt{\left|C_{\gamma}^{12}\right|^{2}+\left|C_{\gamma}^{21}\right|^{2}} \leq 2.45 \times 10^{-10}\left(\frac{\Lambda}{1 \mathrm{TeV}}\right)^{2} \sqrt{\frac{\mathrm{Br}[\mu \rightarrow e \gamma]}{5.7 \times 10^{-13}}} \\
& \sqrt{\left|C_{\gamma}^{13}\right|^{2}+\left|C_{\gamma}^{31}\right|^{2}} \leq 2.35 \times 10^{-6}\left(\frac{\Lambda}{1 \mathrm{TeV}}\right)^{2} \sqrt{\frac{\mathrm{Br}[\tau \rightarrow e \gamma]}{3.3 \times 10^{-8}}} \\
& \sqrt{\left|C_{\gamma}^{23}\right|^{2}+\left|C_{\gamma}^{32}\right|^{2}} \leq 2.71 \times 10^{-6}\left(\frac{\Lambda}{1 \mathrm{TeV}}\right)^{2} \sqrt{\frac{\mathrm{Br}[\tau \rightarrow \mu \gamma]}{4.4 \times 10^{-8}}}
\end{aligned}
$$

Here, the numbers dividing the branching ratios are the current experimental bounds given in table 1. We see that the resulting bounds are very strong, of the order of $10^{-10}$ for $\mu \rightarrow e$ transitions and of the order of $10^{-6}$ for $\tau \rightarrow \mu, e$ transitions for NP at the $\mathrm{TeV}$ scale. This means that, even though in a renormalizable theory of NP $C_{\gamma}^{f i}$ can only be induced at the loop level, an additional suppression mechanism is needed (especially for $\mu \rightarrow e \gamma$ ) in order the make $\mathrm{TeV}$-scale NP compatible with experiment.

Knowing that $C_{\gamma}^{f i}$ must be tiny one can set them to zero in order to constrain other Wilson coefficients using the bounds from the $\ell_{i} \rightarrow \ell_{f} \ell_{f} \bar{\ell}_{f}$ decay rates. Here we find (again 
normalizing the branching ratios to current limits listed in table 2):

$$
\begin{aligned}
& C_{\text {неее }} \leq 3.29 \times 10^{-5}\left(\frac{\Lambda}{1 \mathrm{TeV}}\right)^{2} \sqrt{\frac{\mathrm{Br}[\mu \rightarrow \text { eеe }]}{1 \times 10^{-12}}},
\end{aligned}
$$

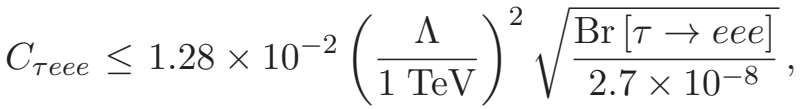

$$
\begin{aligned}
& C_{\tau \mu \mu \mu} \leq 1.13 \times 10^{-2}\left(\frac{\Lambda}{1 \mathrm{TeV}}\right)^{2} \sqrt{\frac{\operatorname{Br}[\tau \rightarrow \mu \mu \mu]}{2.1 \times 10^{-8}}},
\end{aligned}
$$

with $C_{\ell_{i} \ell_{f} \ell_{f} \ell_{f}}$ given by

$$
\begin{aligned}
C_{\ell_{i} \ell_{f} \ell_{f} \ell_{f}}= & \left(2\left|C_{\ell \ell}^{f i f f}-0.54\left(C_{\varphi \ell}^{(1) f i}+C_{\varphi \ell}^{(3) f i}\right)\right|^{2}+2\left|C_{e e}^{f i f f}+0.46 C_{\varphi e}^{f i}\right|^{2}\right. \\
& \left.+\left|C_{\ell e}^{f i f f}+0.46\left(C_{\varphi \ell}^{(1) f i}+C_{\varphi \ell}^{(3) f i}\right)\right|^{2}+\left|C_{\ell e}^{f f f i}-0.54 C_{\varphi e}^{f i}\right|^{2}\right)^{\frac{1}{2}} .
\end{aligned}
$$

From eq. (6.2) and eq. (6.3) we see that also the Wilson coefficient of the flavor changing 4-lepton and the $Z^{0}$-lepton-lepton vertices must be small for $\Lambda \sim \mathcal{O}(1) \mathrm{TeV}$ : of the order of $10^{-5}$ for $\mu \rightarrow e$ transitions and on the order of $10^{-2}$ for $\tau \rightarrow \mu$ and $\tau \rightarrow e$ transitions. These constraints are less stringent then the ones derived from radiative photon decays in eq. (6.1) but one should keep in mind that unlike $O_{e B}$ and $O_{e W}$, the other operators are not necessarily induced at the loop-level but can already be generated at tree-level.

Also the constraints from $Z^{0} \rightarrow \ell_{f}^{ \pm} \ell_{i}^{\mp}$ can be brought into a form in which one can directly read off the bounds on the Wilson coefficients:

$$
\begin{aligned}
& \sqrt{\left|C_{\varphi \ell}^{(1) 12}+C_{\varphi \ell}^{(3) 12}\right|^{2}+\left|C_{\varphi e}^{12}\right|^{2}+\left|C_{Z}^{12}\right|^{2}+\left|C_{Z}^{21}\right|^{2}} \leq 0.06\left(\frac{\Lambda}{1 \mathrm{TeV}}\right)^{2} \sqrt{\frac{\mathrm{Br}\left[Z^{0} \rightarrow \mu^{ \pm} e^{\mp}\right]}{1.7 \times 10^{-6}}} \\
& \sqrt{\left|C_{\varphi \ell}^{(1) 13}+C_{\varphi \ell}^{(3) 13}\right|^{2}+\left|C_{\varphi e}^{13}\right|^{2}+\left|C_{Z}^{13}\right|^{2}+\left|C_{Z}^{31}\right|^{2}} \leq 0.14\left(\frac{\Lambda}{1 \mathrm{TeV}}\right)^{2} \sqrt{\frac{\mathrm{Br}\left[Z^{0} \rightarrow \tau^{ \pm} e^{\mp}\right]}{9.8 \times 10^{-6}}} \\
& \sqrt{\left|C_{\varphi \ell}^{(1) 23}+C_{\varphi \ell}^{(3) 23}\right|^{2}+\left|C_{\varphi e}^{23}\right|^{2}+\left|C_{Z}^{23}\right|^{2}+\left|C_{Z}^{32}\right|^{2}} \leq 0.16\left(\frac{\Lambda}{1 \mathrm{TeV}}\right)^{2} \sqrt{\frac{\operatorname{Br}\left[Z^{0} \rightarrow \tau^{ \pm} \mu^{\mp}\right]}{1.2 \times 10^{-5}}}
\end{aligned}
$$

These constraints are less stringent than the ones from $\ell_{i} \rightarrow \ell_{f} \ell_{f} \bar{\ell}_{f}$ and $\ell_{i} \rightarrow \ell_{f} \gamma$ but they put bounds on the linear combination $C_{Z}^{f i}$ which is orthogonal to $C_{\gamma}^{f i}$ (see eq. (3.3) and eq. (5.5)), so that using both eq. (6.1) and eq. (6.4) one can independently constrain both $C_{e W}^{f i}$ and $C_{e B}^{f i}$.

Finally, one can give similar simplified expressions for the bounds resulting from the anomalous magnetic moments of charged leptons and from the EDMs. Neglecting small lepton mass ratios and taking into account that some of the Wilson coefficients of the 4- 

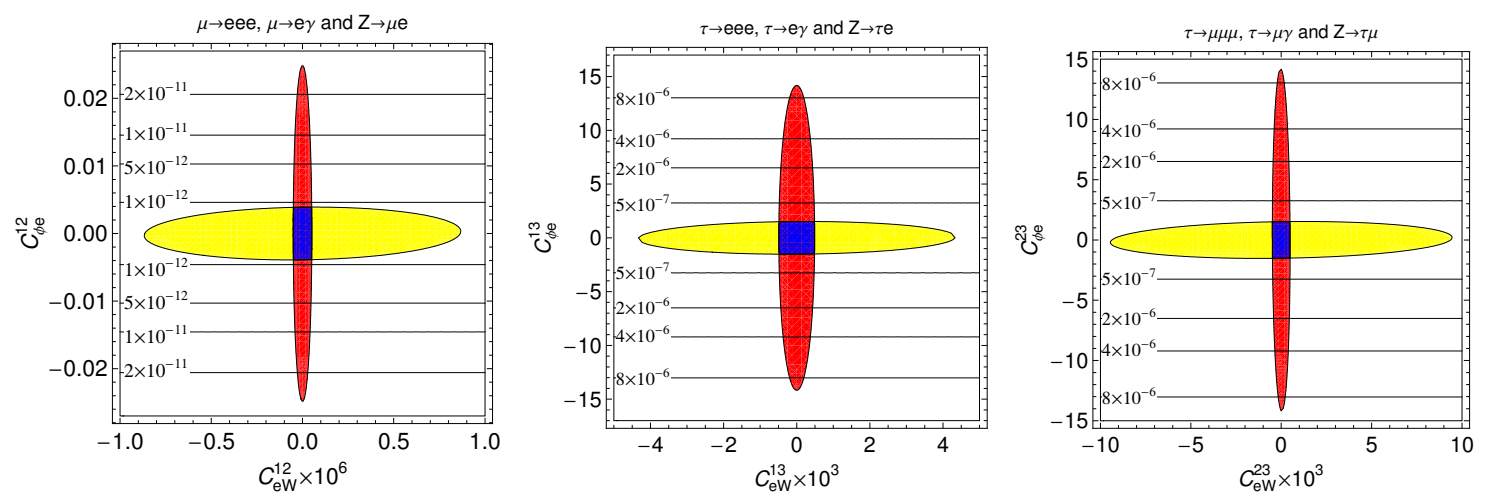

Figure 5. Allowed regions in the $C_{e W}^{f i}-C_{\varphi e}^{f i}$ plane for $\Lambda=10 \mathrm{TeV}$. Yellow (lightest): $\ell_{i} \rightarrow \ell_{f} \ell_{f} \bar{\ell}_{f}$, red (gray): $\ell_{i} \rightarrow \ell_{f} \gamma$. The blue region is allowed by both decay modes simultaneously. The contour lines show the predicted branching ratio for $Z^{0} \rightarrow \ell_{f} \ell_{i}$. Note that in the parameter space plotted, the dependence of $\operatorname{Br}\left[Z^{0} \rightarrow \ell_{f} \ell_{i}\right]$ on $C_{e W}^{f i}$ is very weak.

lepton and the $Z^{0}$-lepton vertices are real in the flavor conserving case we find for the EDMs:

$$
\begin{aligned}
& d_{e}=-2.08 \times 10^{-18} \operatorname{Im}\left[2 \times 10^{-5} C_{\ell e}^{3113}+C_{\gamma}^{11}\right]\left(\frac{1 \mathrm{TeV}}{\Lambda}\right)^{2} e \mathrm{~cm}, \\
& d_{\mu}=-2.08 \times 10^{-18} \operatorname{Im}\left[2 \times 10^{-5} C_{\ell e}^{3223}+C_{\gamma}^{22}\right]\left(\frac{1 \mathrm{TeV}}{\Lambda}\right)^{2} e \mathrm{~cm}, \\
& d_{\tau}=-2.08 \times 10^{-18} \operatorname{Im}\left[C_{\gamma}^{33}\right]\left(\frac{1 \mathrm{TeV}}{\Lambda}\right)^{2} e \mathrm{~cm},
\end{aligned}
$$

and for the anomalous magnetic moments:

$$
\begin{aligned}
& a_{e}=1.17 \times 10^{-6} \operatorname{Re}\left[2 \times 10^{-5} C_{\ell e}^{3113}+C_{\gamma}^{11}\right]\left(\frac{1 \mathrm{TeV}}{\Lambda}\right)^{2}, \\
& a_{\mu}=2.43 \times 10^{-4} \operatorname{Re}\left[2 \times 10^{-5} C_{\ell e}^{3223}+C_{\gamma}^{22}\right]\left(\frac{1 \mathrm{TeV}}{\Lambda}\right)^{2}, \\
& a_{\tau}=4.1 \times 10^{-3} \operatorname{Re}\left[10^{-5} \times\left(1.6 C_{\varphi \ell}^{(1) 33}+2.0 C_{\ell e}^{3333}-1.7\left(C_{\varphi \ell}^{(3) 33}+C_{\varphi e}^{33}\right)\right)+C_{\gamma}^{33}\right]\left(\frac{1 \mathrm{TeV}}{\Lambda}\right)^{2} .
\end{aligned}
$$

Here we kept the loop induced contributions from the $Q_{\ell e}$ and $Q_{\varphi e}$ since they are not (or weakly) constrained from other processes.

In order to illustrate the interplay between different Wilson coefficients in $\ell_{i} \rightarrow \ell_{f} \gamma$ and $\ell_{i} \rightarrow \ell_{f} \ell_{f} \bar{\ell}_{f}$ decays let us consider as an example the dependence of both decays on the Wilson coefficients of the operators $O_{\varphi e}^{f i}$ and $O_{e W}^{f i}$, as shown in figure 5. We see that the regions which respect both the bound from $\ell_{i} \rightarrow \ell_{f} \gamma$ and $\ell_{i} \rightarrow \ell_{f} \ell_{f} \bar{\ell}_{f}$ are very small, especially for $\mu \rightarrow e$ transitions. We also show the predicted branching ratios for $Z^{0} \rightarrow \ell_{f} \ell_{i}$ to illustrate that in this plane indirect limits from the other two processes are currently stronger then the directly measured upper bounds given in table 7 .

Another interesting aspect is the correlation between the radiative lepton decays and the three-body charged lepton decays. In figure 6 we show as an example the ratios 

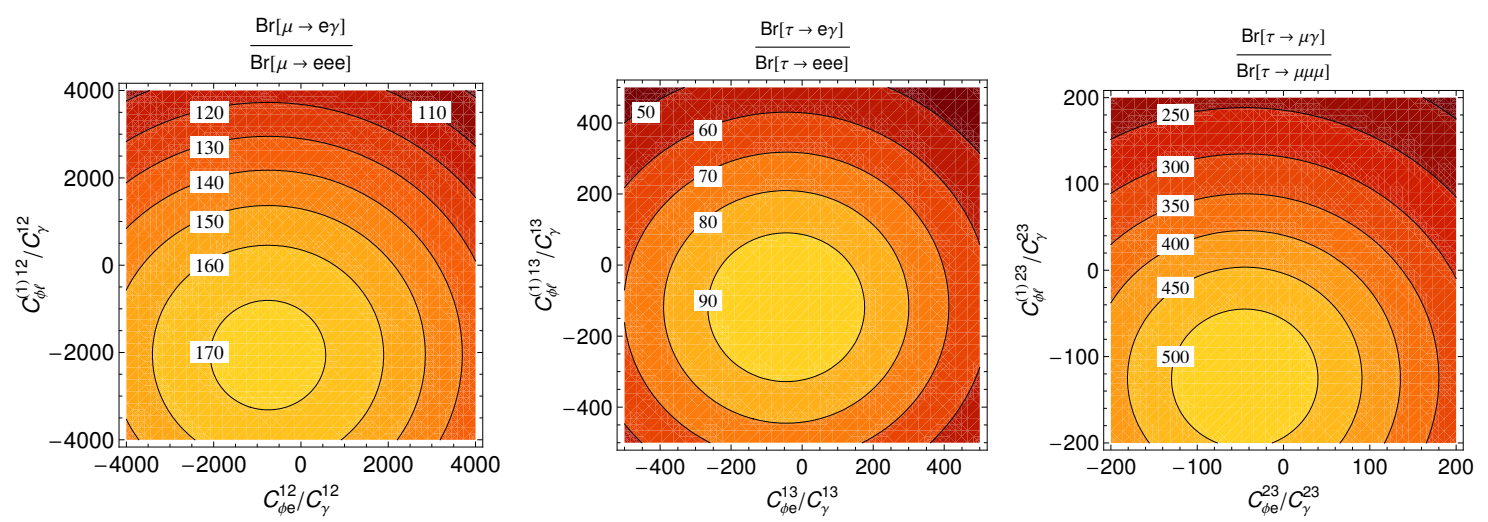

Figure 6. Ratios $\operatorname{Br}\left[\ell_{i} \rightarrow \ell_{f} \gamma\right] / \operatorname{Br}\left[\ell_{i} \rightarrow \ell_{f} \ell_{f} \bar{\ell}_{f}\right]$ in the $\frac{C_{\varphi e}^{f i}}{C_{\gamma}^{f i}}-\frac{C_{\varphi \ell}^{(1)} f i}{C_{\gamma}^{f i}}$ plane (independent of the scale $\Lambda$ of NP).

$\operatorname{Br}\left[\ell_{i} \rightarrow \ell_{f} \gamma\right] / \operatorname{Br}\left[\ell_{i} \rightarrow \ell_{f} \ell_{f} \bar{\ell}_{f}\right]$ as a function of $\frac{C_{\varphi e}^{f i}}{C_{\gamma}^{f i}}$ and $\frac{C_{\varphi \ell}^{(1)} f i}{C_{\gamma}^{f i}}$. Note that such ratios are independent of the scale $\Lambda$ of NP and depend only on the ratios of Wilson coefficients. Thus, given a specific model, one can determine the branching ratio for one process in terms of the other one independently of the scale of new physics and also of other possible cancellations of NP model parameters which can occur in the ratios $\frac{C_{\varphi}^{f i}}{C_{\gamma}^{f i}}$ and $\frac{C_{\varphi \ell}^{(1)} f i}{C_{\gamma}^{f i}}$. As known in the literature, the ratio of both decay rates in case in which only $C_{\gamma}^{f i}$ is non-zero depends solely on SM parameters and is given by $1 /\left(\frac{\alpha}{3 \pi}\left(\log \frac{m_{f}^{2}}{m_{i}^{2}}-\frac{11}{4}\right)\right)$ (which corresponds to points $(0,0)$ in figure 6$)$. From figure 6 one can see that contributions from $C_{\varphi e}^{f i}$ and $C_{\varphi \ell}^{(1) f i}$ can only slightly enhance but more significantly suppress this ratio. This is important from the point of view of planned new experiments searching for $\mu \rightarrow$ eee with increased sensitivity.

As observed in section 5, processes involving photon and $Z^{0}$ couplings to leptons constrain "orthogonal" combinations of the Wilson coefficients of the operators $O_{e B}$ and $O_{e W}$. Thus, using a suitable pair of measurements, one can obtain absolute upper bounds on each of $C_{e B}$ and $C_{e W}$. An example of such an exclusion is shown in the left panel of figure 7: the bound on the radiative decay $\tau \rightarrow \mu \gamma$ strongly correlates the allowed values for $C_{e B}$ and $C_{e W}$ values to a thin straight belt, while $Z^{0} \rightarrow \tau \mu$ bound cuts the length of this belt to a wider but finite compartment.

Concerning flavor diagonal transitions we can correlate the anomalous magnetic moments to the corresponding $Z^{0} \rightarrow \ell \ell$ decays. For the electron and the muon the constraints from the anomalous magnetic moments are so strong that no sizable effects of NP in $Z^{0} \rightarrow e e$ or $Z^{0} \rightarrow \mu \mu$ are possible. However, for the tau lepton the constraints on NP generated terms from $Z^{0} \rightarrow \tau \tau$ and from the anomalous magnetic moment are not that different. The allowed region in the $C_{e W}^{33}-C_{e B}^{33}$ plane is shown in the middle plot of figure 7 . In order to obtain these constraint we used $\operatorname{Br}\left[Z^{0} \rightarrow \tau \tau\right]=(3.370 \pm 0.008) \%$ [1] and included radiative corrections into our tree-level expression for $Z^{0} \rightarrow \ell_{f} \ell_{i}$, eq. (5.1), multiplying it by a correction factor $\operatorname{Br}\left[Z^{0} \rightarrow \tau \tau\right]_{\mathrm{SM}} / \operatorname{Br}\left[Z^{0} \rightarrow \tau \tau\right]_{\text {tree }}$ where $\operatorname{Br}\left[Z^{0} \rightarrow \tau \tau\right]_{\mathrm{SM}}$ 

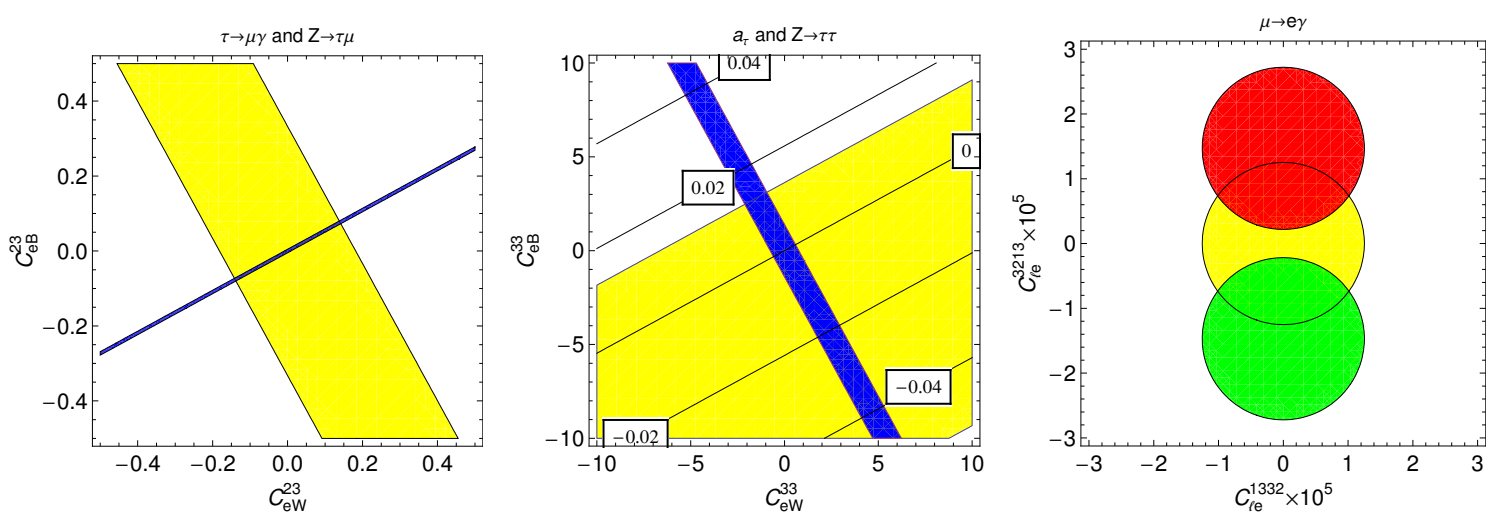

Figure 7. Left: allowed regions from $\operatorname{Br}\left[Z^{0} \rightarrow \tau \mu\right]$ (yellow) and $\operatorname{Br}[\tau \rightarrow \mu \gamma]$ (blue) in the $C_{e W}^{23}-C_{e B}^{23}$ plane for $\Lambda=1 \mathrm{TeV}$. Middle: correlations between the anomalous magnetic moment of the $\tau$ lepton and $Z^{0} \rightarrow \tau \tau$. Yellow (light grey): region allowed by the $a_{\tau}$, blue (dark grey): region allowed by $Z^{0} \rightarrow \tau \tau$. The contour lines indicate the value of $a_{\tau}$ for $\Lambda=1 \mathrm{TeV}$. Right: allowed regions from $\operatorname{Br}[\mu \rightarrow e \gamma]$ in the $C_{\ell e}^{1332}-C_{\ell e}^{3213}$ plane for $\Lambda=1 \mathrm{TeV}$ and different values of $C_{e W}^{12}$. Yellow: $C_{e W}^{12}=0$, red: $C_{e W}^{12}=6 \times 10^{-8}$, green: $C_{e W}^{12}=-6 \times 10^{-8}$.

includes radiative corrections and can be found in ref. [1]. We also find that the precision of $Z^{0} \rightarrow \ell_{j} \bar{\ell}_{j}$ decay width measurements limit the sizes of $C_{\varphi \ell}^{(1) j j}, C_{\varphi \ell}^{(3) j j}$ and $C_{\varphi e}^{j j}$ Wilson coefficients so stringently that no sizable effects in the corresponding anomalous magnetic moments are possible for any lepton flavor.

Another interesting aspect is that one can constrain some of the 4-lepton contact terms by using only the radiative lepton decays. This is possible because the 4-lepton operator $O_{\ell e}$ affects the $\ell_{i} \rightarrow \ell_{f} \gamma$ amplitude at the 1-loop level, as calculated in section 3.1. Once the values of Wilson coefficients defining the photon coupling $C_{\gamma}$ are fixed, the bounds on the 4-lepton couplings can be fairly strong - as illustrated in example in the right panel of figure 7. There we see that the bounds on $C_{\ell e}^{1332}$ and $C_{\ell e}^{3213}$ from $\mu \rightarrow e \gamma$ for $\Lambda=1 \mathrm{TeV}$ are $\mathcal{O}\left(10^{-5}\right)$. Note that these coefficients (with double $\tau$ flavor index) cannot be constrained from any other process considered in this article.

\section{Conclusions}

In these article we calculated the expressions for several theoretically important and experimentally well constrained lepton flavor violating processes within the Standard Model extended with the most general set of effective LFV operators of dimension-6 invariant under the SM gauge group. We computed the complete set of 1-loop contributions (to the leading order in $m_{\ell} / m_{W}$ ) to the radiative lepton decays $\ell_{i} \rightarrow \ell_{f} \gamma$ and to the related electric dipole moments and anomalous magnetic moments of charged leptons (see table 6). We also obtained the full expression for the 3-body charged lepton decay rates $\ell_{i} \rightarrow \ell_{j} \ell_{k} \ell_{l}$ (eqs. (4.5)-(4.9)) and for the flavor violating $Z^{0} \rightarrow l_{f} \bar{l}_{i}$ decays taking into account all possible tree-level contributions.

The predictions for all processes are given in terms of Wilson coefficients of the effective operators, automatically assuring that the final results are gauge-invariant (which we con- 
firmed explicitly in our calculation) and that all relevant contributions are included. The derived expressions allow to obtain model-independent bounds on the Wilson coefficients of LFV operators, which can be later easily compared to their values calculated within specific UV complete extensions of the SM.

To facilitate the comparison, we included in section 6 approximate numerical formulae directly relating the Wilson coefficients to current experimental upper bounds on the discussed processes (eq. (6.1)-eq. (6.6)). We show that bounds on the effective LFV couplings are already very strong if the scale of $\mathrm{NP}$ is low, $\mathcal{O}(1) \mathrm{TeV}$, and weaken proportionally to the square of NP scale. We also illustrated possible correlations between Wilson coefficients of various dimension- 6 operators and showed that the loop contributions to $\ell_{i} \rightarrow \ell_{f} \gamma$ decays are capable to constrain 4-lepton operators which would be unbounded otherwise.

\section{Acknowledgments}

The authors would like to thank Stefan Pokorski for suggesting the subject of this article and helpful discussions. A.C. is grateful to Christoph Greub for his help and proofreading the article. We also thank Marco Giovanni Pruna and Adrian Signer for useful discussions. A.C. is supported by the Swiss National Science Foundation (SNF). The work of S.N. has been suported by the Marie Curie Initial Training Network of the EU Seventh Framework Programme under contract number PITN-GA-2009-237920-UNILHC. She thanks the CERN Theory Division for the hospitality during the stay there. The work of J.R. is supported by the National Science Center in Poland under the research grants DEC-2011/01/M/ST2/02466 and DEC-2012/05/B/ST2/02597.

\section{A Feynman rules and vector/scalar form-factors}

We summarize below the Feynman rules arising from the dimension- 6 operators after the electroweak symmetry breaking. $i, i_{1}, i_{2}$ and $f, f_{1}, f_{2}$ denote the flavor indices of incoming and outgoing leptons, respectively. We list only the vertices actually used in our tree level or 1-loop calculations. For completeness we also include few necessary purely SM couplings.

\section{A.1 Feynman rules involving gauge and Goldstone bosons}

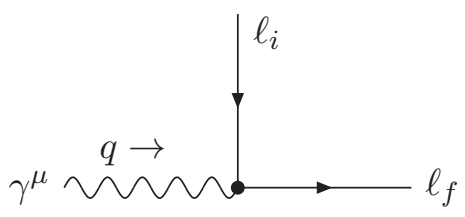

$$
\begin{gathered}
i\left(e \gamma^{\mu} \delta^{f i}+i \sigma^{\mu \nu}\left[C_{\gamma L}^{f i} P_{L}+C_{\gamma R}^{f i} P_{R}\right] q_{\nu}\right) \\
C_{f i}^{\gamma R}=C_{f i}^{\gamma L \star}=\frac{v \sqrt{2}}{\Lambda^{2}}\left(c_{W} C_{e B}^{f i}-s_{W} C_{e W}^{f i}\right)
\end{gathered}
$$


$Z^{\mu} \sim^{\ell_{i}} \rightarrow \ell_{f}$
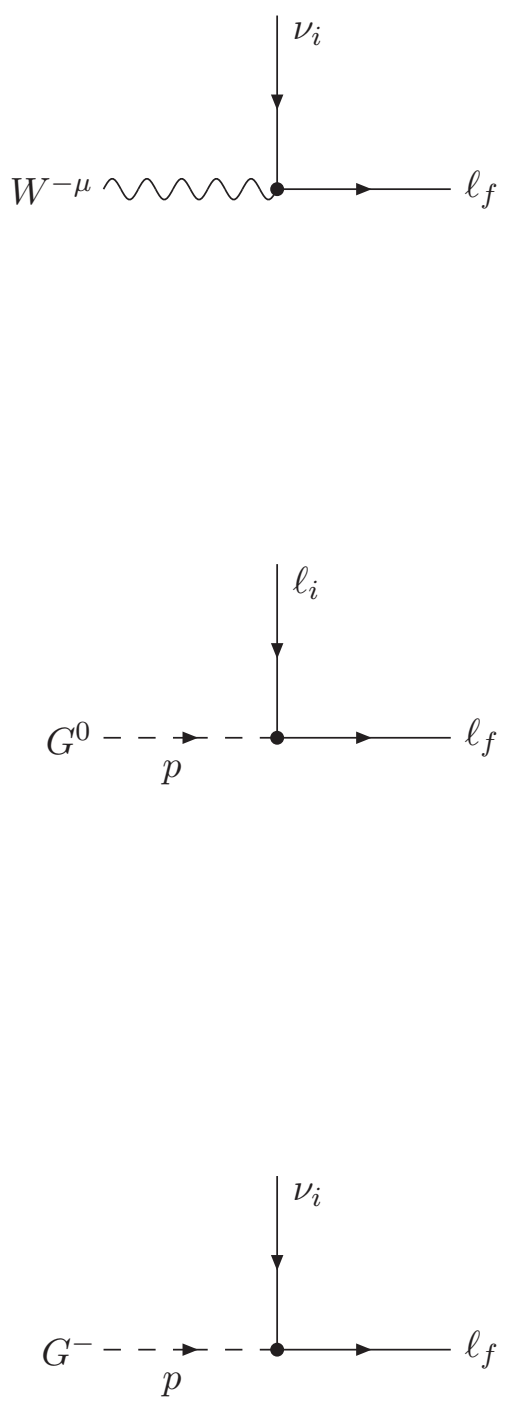

$$
i\left(\gamma^{\mu}\left[\Gamma_{f i}^{Z L} P_{L}+\Gamma_{f i}^{Z R} P_{R}\right]+i \sigma^{\mu \nu}\left[C_{f i}^{Z L} P_{L}+C_{f i}^{Z R} P_{R}\right] q_{\nu}\right)
$$

$$
\begin{aligned}
\Gamma_{f i}^{Z L} & =\frac{e}{2 s_{W} c_{W}}\left(\frac{v^{2}}{\Lambda^{2}}\left(C_{\phi \ell}^{(1) f i}+C_{\phi \ell}^{(3) f i}\right)+\left(1-2 s_{W}^{2}\right) \delta_{f i}\right) \\
\Gamma_{f i}^{Z R} & =\frac{e}{2 s_{W} c_{W}}\left(\frac{v^{2}}{\Lambda^{2}} C_{\phi e}^{f i}-2 s_{W}^{2} \delta_{f i}\right) \\
C_{f i}^{Z R} & =C_{i f}^{Z L \star}=-\frac{v \sqrt{2}}{\Lambda^{2}}\left(s_{W} C_{e B}^{f i}+c_{W} C_{e W}^{f i}\right)
\end{aligned}
$$

$i \Gamma_{f j}^{W L} V_{j i}^{P M N S} \gamma^{\mu} P_{L}$

$$
\Gamma_{f j}^{W L}=-\frac{e}{\sqrt{2} s_{W}}\left(\frac{v^{2}}{\Lambda^{2}} C_{\phi \ell}^{(3) f j}+\delta_{f j}\right)
$$

$-\left(\left(\not p \Gamma_{f i}^{G^{0} L}+\frac{1}{v} \delta_{f i} m_{\ell_{i}}\right) P_{L}\right.$

$\left.+\left(\not p \Gamma_{f i}^{G^{0} R}-\frac{1}{v} \delta_{f i} m_{\ell_{i}}\right) P_{R}\right)$

$\Gamma_{f i}^{G^{0} L}=\frac{v}{\Lambda^{2}}\left(C_{\phi \ell}^{(1) f i}+C_{\phi \ell}^{(3) f i}\right)$

$\Gamma_{f i}^{G^{0} R}=\frac{v}{\Lambda^{2}} C_{\phi e}^{f i}$

$$
\begin{aligned}
& i\left(\Gamma_{f j}^{G^{-} L} \not p-\frac{\sqrt{2}}{v} \delta_{f j} m_{\ell_{f}}\right) V_{j i}^{P M N S} P_{L} \\
& \Gamma_{f j}^{G^{-} L}=-\frac{v \sqrt{2}}{\Lambda^{2}} C_{\phi l}^{(3) f j}
\end{aligned}
$$



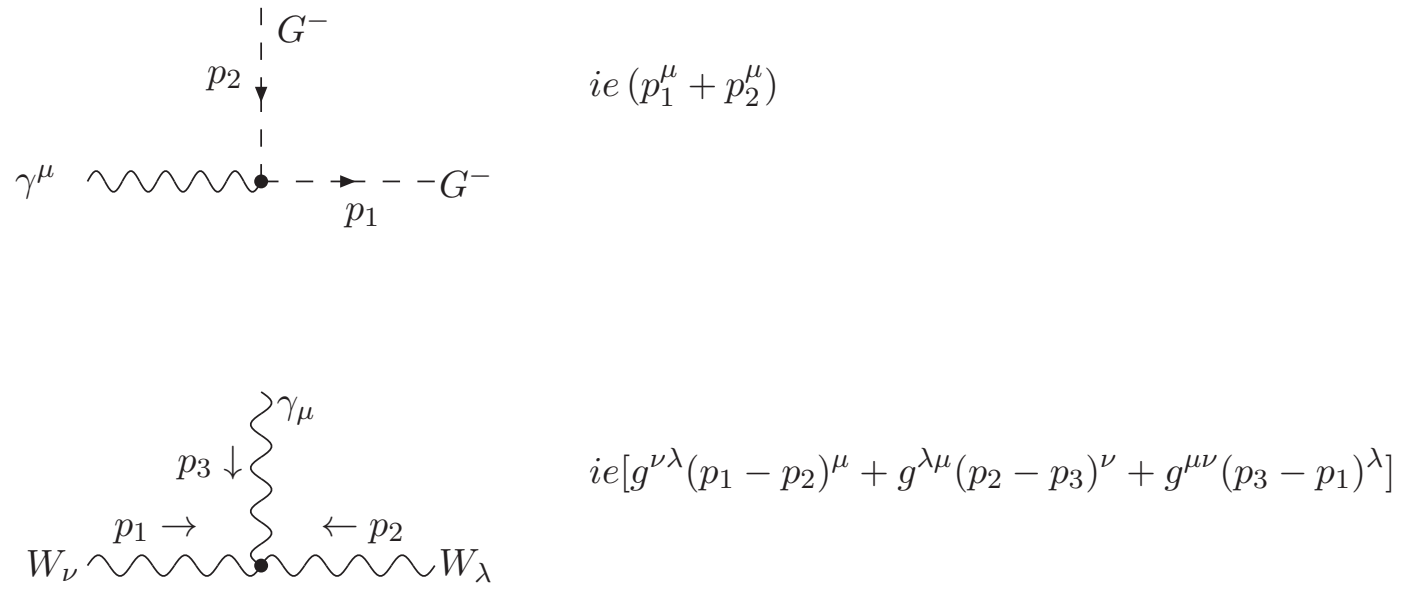

$$
\begin{gathered}
i \Gamma_{f j}^{G \gamma L} V_{j i}^{P M N S} \gamma^{\mu} P_{L} \\
\Gamma_{f j}^{G \gamma L}=-\frac{e v \sqrt{2}}{\Lambda^{2}} C_{\phi l}^{(3) f j}
\end{gathered}
$$

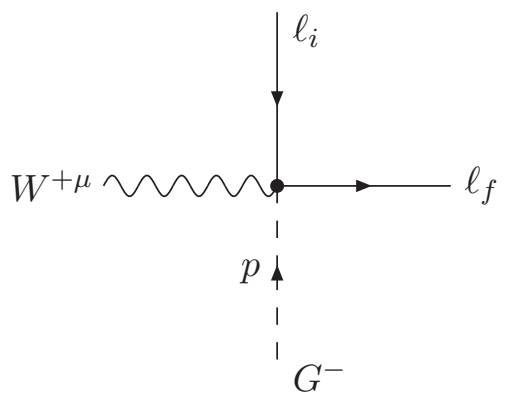

$$
\begin{aligned}
& i \gamma^{\mu}\left[\Gamma_{f i}^{G W L} P_{L}+\Gamma_{f i}^{G W R} P_{R}\right] \\
& \Gamma_{f i}^{G W L}=-\frac{e v}{\Lambda^{2} s_{W}} C_{\phi \ell}^{(1) f i} \\
& \Gamma_{f i}^{G W R}=-\frac{e v}{\Lambda^{2} s_{W}} C_{\phi e}^{f i}
\end{aligned}
$$

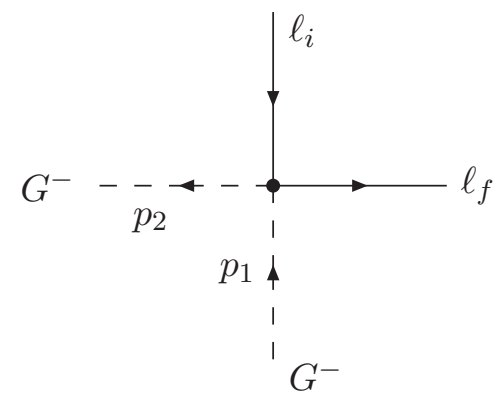

$$
\begin{aligned}
& i\left(\not p_{1}+\not p_{2}\right)\left[\Gamma_{f i}^{G G L} P_{L}+\Gamma_{f i}^{G G R} P_{R}\right] \\
& \Gamma_{f i}^{G G L}=-\frac{1}{\Lambda^{2}}\left(C_{\phi l}^{(1) f i}-C_{\phi \ell}^{(3) f i}\right) \\
& \Gamma_{f i}^{G G R}=-\frac{1}{\Lambda^{2}} C_{\phi e}^{f i}
\end{aligned}
$$




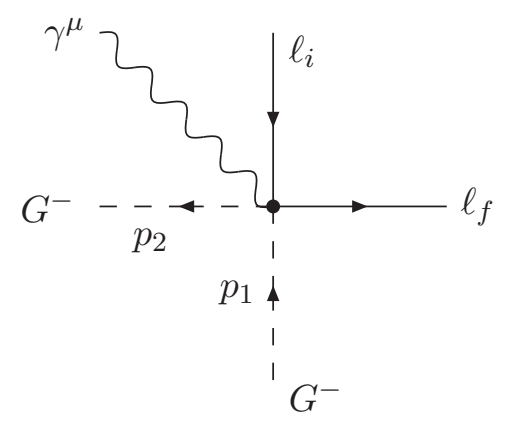

$$
\begin{gathered}
i \gamma^{\mu}\left(\Gamma_{f i}^{G G \gamma L} P_{L}+\Gamma_{f i}^{G G \gamma R} P_{R}\right) \\
\Gamma_{f i}^{G G \gamma L}=-\frac{2 e}{\Lambda^{2}}\left(C_{\phi \ell}^{(1) f i}-C_{\phi \ell}^{(3) f i}\right) \\
\Gamma_{f i}^{G G \gamma R}=-\frac{2 e}{\Lambda^{2}} C_{\phi e}^{f i}
\end{gathered}
$$

\section{A.2 Feynman rules for 4-fermion operators}

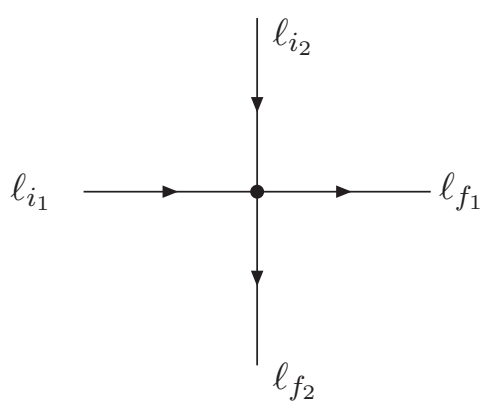

$$
\begin{aligned}
& \frac{i}{\Lambda^{2}}\left[C_{\ell \ell}^{f_{1} i_{1} f_{2} i_{2}}\left(\gamma^{\mu} P_{L}\right)_{f_{1} i_{1}}\left(\gamma_{\mu} P_{L}\right)_{f_{2} i_{2}}\right. \\
& +C_{e e}^{f_{1} i_{1} f_{2} i_{2}}\left(\gamma^{\mu} P_{R}\right)_{f_{1} i_{1}}\left(\gamma_{\mu} P_{R}\right)_{f_{2} i_{2}} \\
& \left.+C_{\ell e}^{f_{1} i_{1} f_{2} i_{2}}\left(\gamma^{\mu} P_{L}\right)_{f_{1} i_{1}}\left(\gamma_{\mu} P_{R}\right)_{f_{2} i_{2}}\right]
\end{aligned}
$$

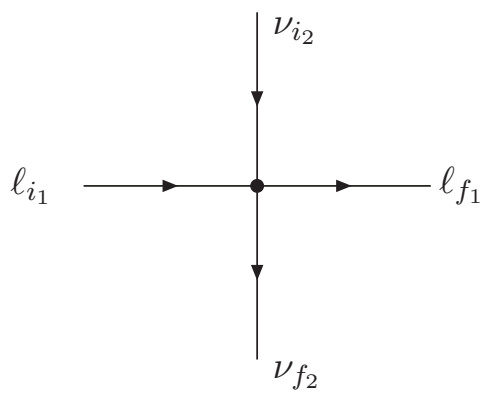

$$
\begin{aligned}
& \frac{i}{\Lambda^{2}}\left[C_{\ell \ell}^{f_{1} i_{1} f_{2} i_{2}}\left(\gamma^{\mu} P_{L}\right)_{i_{1} f_{1}}\left(\gamma_{\mu} P_{L}\right)_{f_{2} i_{2}}\right. \\
& \left.+2 \operatorname{Re}\left(C_{\ell e}^{f_{1} i_{1} f_{2} i_{2}}\right)\left(\gamma^{\mu} P_{L}\right)_{f_{1} i_{1}}\left(\gamma_{\mu} P_{R}\right)_{f_{2} i_{2}}\right]
\end{aligned}
$$

$$
\frac{i}{\Lambda^{2}}\left[\left(C_{\ell q}^{(1) f_{1} i_{1} f_{2} i_{2}}-C_{\ell q}^{(3) f_{1} i_{1} f_{2} i_{2}}\right)\left(\gamma^{\mu} P_{L}\right)_{i_{1} f_{1}}\left(\gamma_{\mu} P_{L}\right)_{f_{2} i_{2}}\right.
$$$$
+C_{\ell u}^{f_{1} i_{1} f_{2} i_{2}}\left(\gamma^{\mu} P_{L}\right)_{f_{1} i_{1}}\left(\gamma_{\mu} P_{R}\right) f_{2} i_{2}
$$

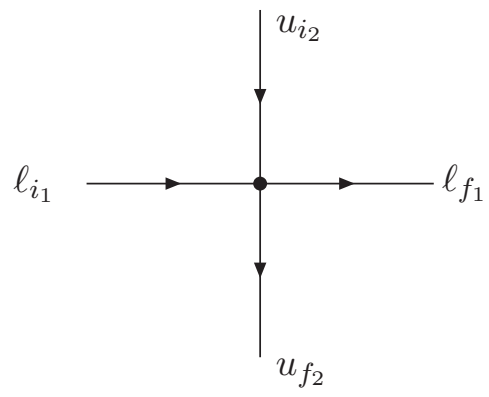

$$
+C_{e q}^{f_{1} i_{1} f_{2} i_{2}}\left(\gamma^{\mu} P_{R}\right)_{f_{1} i_{1}}\left(\gamma_{\mu} P_{L}\right)_{f_{2} i_{2}}
$$$$
+C_{e u}^{f_{1} i_{1} f_{2} i_{2}}\left(\gamma^{\mu} P_{R}\right)_{f_{1} i_{1}}\left(\gamma_{\mu} P_{R}\right) f_{2} i_{2}
$$$$
-C_{\text {lequ }}^{(1) f_{1} i_{1} f_{2} i_{2}}\left(P_{R}\right)_{f_{1} i_{1}}\left(P_{R}\right)_{f_{2} i_{2}}
$$$$
-C_{\ell e q u}^{(1) i_{1} f_{1} i_{2} f_{2} \star}\left(P_{L}\right)_{f_{1} i_{1}}\left(P_{L}\right)_{f_{2} i_{2}}
$$$$
-C_{\text {lequ }}^{(3) f_{1} i_{1} f_{2} i_{2}}\left(\sigma^{\mu \nu} P_{R}\right)_{f_{1} i_{1}}\left(\sigma_{\mu \nu} P_{R}\right)_{f_{2} i_{2}}
$$$$
\left.-C_{\text {lequ }}^{(3) i_{1} f_{1} i_{2} f_{2} \star}\left(\sigma^{\mu \nu} P_{L}\right)_{f_{1} i_{1}}\left(\sigma_{\mu \nu} P_{L}\right)_{f_{2} i_{2}}\right]
$$ 


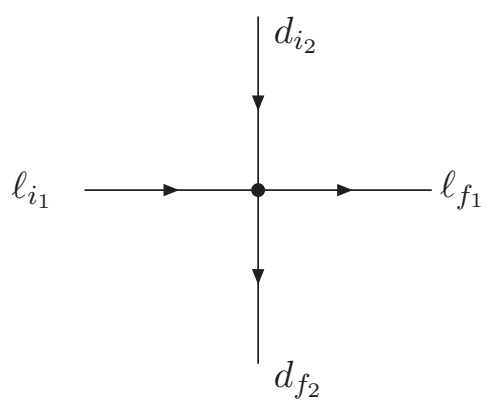

$$
\begin{aligned}
& \frac{i}{\Lambda^{2}}\left[\left(C_{\ell q}^{(1) f_{1} i_{1} f_{2} i_{2}}+C_{\ell q}^{(3) f_{1} i_{1} f_{2} i_{2}}\right)\left(\gamma^{\mu} P_{L}\right)_{i_{1} f_{1}}\left(\gamma_{\mu} P_{L}\right)_{f_{2} i_{2}}\right. \\
& +C_{\ell d}^{f_{1} i_{1} f_{2} i_{2}}\left(\gamma^{\mu} P_{L}\right)_{f_{1} i_{1}}\left(\gamma_{\mu} P_{R}\right)_{f_{2} i_{2}} \\
& +C_{e q}^{f_{1} i_{1} f_{2} i_{2}}\left(\gamma^{\mu} P_{R}\right)_{f_{1} i_{1}}\left(\gamma_{\mu} P_{L}\right)_{f_{2} i_{2}} \\
& +C_{e d}^{f_{1} i_{1} f_{2} i_{2}}\left(\gamma^{\mu} P_{R}\right)_{f_{1} i_{1}}\left(\gamma_{\mu} P_{R}\right)_{f_{2} i_{2}} \\
& \left.+C_{\ell e d q}^{f_{1} i_{1} f_{2} i_{2}}\left(P_{R}\right)_{f_{1} i_{1}}\left(P_{L}\right)_{f_{2} i_{2}}+C_{\ell e d q}^{i_{1} f_{1} i_{2} f_{2} \star}\left(P_{L}\right)_{f_{1} i_{1}}\left(P_{R}\right)_{f_{2} i_{2}}\right]
\end{aligned}
$$

\section{A.3 Vector and scalar form-factors contributing to off-shell $\ell_{i} \rightarrow \ell_{f} \gamma^{*}$ ampli- tude}

Gauge invariance requires that $F_{V L}$ and $F_{V R}$ ("vector") form-factors vanish for the on-shell external particles. Thus, expressions for them must be proportional to the momentum of the outgoing photon and they do not contribute to $\ell_{i} \rightarrow \ell_{f} \gamma$ decay rate. The "scalar" formfactors $F_{S L}$ and $F_{S R}$ does not need to vanish on-shell, but they also cancel out from this amplitude after contracting with the photon polarization vector. Still, those form-factors can enter the expressions for the more complicated processes. Thus, we list them below, again splitted into groups of contributions within which the vector form-factors vanish in the on-shell limit. Note that some of them are infinite and require renormalization.

We give only expressions for left scalar form-factor $F_{S L}$ - the right one can be obtained from $F_{S L}$ by changing the sign and exchanging the external fermion masses, i.e.:

$$
F_{S R}=-F_{S L}\left(m_{i} \leftrightarrow m_{f}\right)
$$

$Z^{0}$ group - diagrams $3 \mathrm{a}, 2 \mathrm{a}\left(Z^{0}\right)$ :

$$
\begin{aligned}
& F_{V L}^{Z f i}=\frac{2 e\left(1-2 s_{W}^{2}\right) Q^{2}}{9(4 \pi)^{2}}\left(C_{\varphi \ell}^{(1) f i}+C_{\varphi l}^{(3) f i}\right)\left(1-6 \log \frac{m_{i} m_{f}}{M_{Z}^{2}}\right) \\
& F_{V R}^{Z f i}=-\frac{4 e s_{W}^{2} Q^{2}}{9(4 \pi)^{2}} C_{\varphi e}^{f i}\left(1-6 \log \frac{m_{i} m_{f}}{M_{Z}^{2}}\right) \\
& F_{S L}^{Z f i}=\frac{2 e}{9(4 \pi)^{2}}\left[m_{f}\left(1-2 s_{W}^{2}\right)\left(C_{\varphi \ell}^{(1) f i}+C_{\varphi l}^{(3) f i}\right)+2 m_{i} s_{W}^{2} C_{\varphi e}^{f i}\right]\left(1-6 \log \frac{m_{i} m_{f}}{M_{Z}^{2}}\right)
\end{aligned}
$$

$W G$ group - diagrams 3c,d,e,i,j,k,l, $2 \mathrm{~b}(W), \mathrm{c}$ and photon-Goldstone boson self-energy:

$$
\begin{aligned}
F_{V L}^{W G f i}= & -\frac{2 e Q^{2}}{9(4 \pi)^{2}}\left[16 C_{\varphi l}^{(3) f i}+6 c_{W}^{2}\left(C_{\varphi \ell}^{(1) f i}+C_{\varphi l}^{(3) f i}\right)\right. \\
& \left.+3 c_{W}^{2}\left(15 C_{\varphi \ell}^{(1) f i}+16 C_{\varphi l}^{(3) f i}\right)\left(\Delta-\log \frac{M_{W}^{2}}{\mu^{2}}\right)\right] \\
F_{V R}^{W G f i}= & -\frac{2 e c_{W}^{2} Q^{2}}{3(4 \pi)^{2}} C_{\varphi e}^{f i}\left[2+15\left(\Delta-\log \frac{M_{W}^{2}}{\mu^{2}}\right)\right]
\end{aligned}
$$




$$
\begin{aligned}
F_{S L}^{W G f i}= & \frac{e}{9(4 \pi)^{2}}\left[12 c_{W}^{2}\left(m_{i} C_{\varphi e}^{f i}-m_{f}\left(C_{\varphi \ell}^{(1) f i}+C_{\varphi l}^{(3) f i}\right)\right)-32 m_{f} C_{\varphi l}^{(3) f i}\right. \\
& \left.+3\left(15 c_{W}^{2}\left(m_{i} C_{\varphi e}^{f i}-m_{f}\left(C_{\varphi \ell}^{(1) f i}+C_{\varphi l}^{(3) f i}\right)\right)-2 m_{f} C_{\varphi l}^{(3) f i}\right)\left(\Delta-\log \frac{M_{W}^{2}}{\mu^{2}}\right)\right]
\end{aligned}
$$

$G^{0}$ group — diagrams $3 \mathrm{~b}, 2 \mathrm{a}\left(G^{0}\right)$ :

$$
F_{V L}^{G^{0}} f i=F_{V R}^{G^{0}}{ }^{f i}=F_{S L}^{G^{0}}{ }^{f i}=F_{S R}^{G^{0}} f i=0
$$

$G^{ \pm}$group - diagrams 3f,g,h, $2 \mathrm{~b}\left(G^{ \pm}\right)$:

$$
F_{V L}^{G^{ \pm} f i}=F_{V R}^{G^{ \pm}} f i=F_{S L}^{G^{ \pm}} f i=F_{S R}^{G^{ \pm}} f i=0
$$

$4 l$ group - contact 4-lepton diagrams 3n, 2e:

$$
\begin{aligned}
& F_{V L}^{4 \ell f i}=-\frac{2 e Q^{2}}{3(4 \pi)^{2}} \sum_{j=1}^{3}\left(2 C_{\ell \ell}^{f i j j}+C_{\ell e}^{f i j j}\left(\Delta-\log \frac{m_{\ell_{j}}^{2}}{\mu^{2}}\right)\right) \\
& F_{V R}^{4 \ell f i}=-\frac{2 e Q^{2}}{3(4 \pi)^{2}} \sum_{j=1}^{3}\left(2 C_{e e}^{f i j j}+C_{\ell e}^{j j f i}\left(\Delta-\log \frac{m_{\ell_{j}}^{2}}{\mu^{2}}\right)\right) \\
& F_{S L}^{4 \ell f i}=-\frac{2 e}{3(4 \pi)^{2}} \sum_{j=1}^{3}\left(2 C_{\ell \ell}^{f i j j} m_{f}-2 C_{e e}^{f i j j} m_{i}-\left(C_{\ell e}^{j j f i} m_{i}-C_{\ell e}^{f i j j} m_{f}\right)\left(\Delta-\log \frac{m_{\ell_{j}}^{2}}{\mu^{2}}\right)\right)
\end{aligned}
$$

$4 f$ group - contact 4-lepton and 2-lepton-2-quark diagrams 3m, 2d:

$$
\begin{aligned}
F_{V L}^{4 f f i}= & \frac{4 e Q^{2}}{9(4 \pi)^{2}} \sum_{j=1}^{3}\left(C_{\ell q}^{(1) f i j j}-C_{\ell q}^{(3) f i j j}+C_{\ell u}^{f i j j}\right)\left(\Delta-\log \frac{m_{u_{j}}^{2}}{\mu^{2}}\right) \\
& -\frac{2 e Q^{2}}{9(4 \pi)^{2}} \sum_{j=1}^{3}\left(C_{\ell q}^{(1) f i j j}+C_{\ell q}^{(3) f i j j}+C_{\ell d}^{f i j j}\right)\left(\Delta-\log \frac{m_{d_{j}}^{2}}{\mu^{2}}\right) \\
F_{V R}^{4 f f i}= & \frac{4 e Q^{2}}{9(4 \pi)^{2}} \sum_{j=1}^{3}\left(C_{e q}^{f i j j}+C_{e u}^{(3) f i j j}\right)\left(\Delta-\log \frac{m_{u_{j}}^{2}}{\mu^{2}}\right) \\
& -\frac{2 e Q^{2}}{9(4 \pi)^{2}} \sum_{j=1}^{3}\left(C_{e q}^{f i j j}+C_{e d}^{(3) f i j j}\right)\left(\Delta-\log \frac{m_{d_{j}}^{2}}{\mu^{2}}\right) \\
F_{S L}^{4 f f i}= & \frac{4 e}{9(4 \pi)^{2}} \sum_{j=1}^{3}\left(m_{f}\left(C_{\ell q}^{(1) f i j j}-C_{\ell q}^{(3) f i j j}+C_{\ell u}^{f i j j}\right)-m_{i}\left(C_{e q}^{f i j j}+C_{e u}^{(3) f i j j}\right)\right) \\
& \times\left(\Delta-\log \frac{m_{u_{j}}^{2}}{\mu^{2}}\right) \\
& -\frac{2 e}{9(4 \pi)^{2}} \sum_{j=1}^{3}\left(m_{f}\left(C_{\ell q}^{(1) f i j j}+C_{\ell q}^{(3) f i j j}+C_{\ell d}^{f i j j}\right)-m_{i}\left(C_{e q}^{f i j j}+C_{e d}^{(3) f i j j}\right)\right) \\
& \times\left(\Delta-\log \frac{m_{d_{j}}^{2}}{\mu^{2}}\right)
\end{aligned}
$$


Open Access. This article is distributed under the terms of the Creative Commons Attribution License (CC-BY 4.0), which permits any use, distribution and reproduction in any medium, provided the original author(s) and source are credited.

\section{References}

[1] Particle Data Group collaboration, J. Beringer et al., Review of Particle Physics (RPP), Phys. Rev. D 86 (2012) 010001 [InSPIRE].

[2] T. Appelquist and J. Carazzone, Infrared Singularities and Massive Fields, Phys. Rev. D 11 (1975) 2856 [inSPIRE].

[3] S. Weinberg, The Quantum theory of fields. Vol. 1: Foundations, Cambridge University Press, Cambridge U.K. (1995).

[4] A.M. Baldini et al., MEG Upgrade Proposal, arXiv:1301.7225 [INSPIRE].

[5] BABAR collaboration, B. Aubert et al., Searches for Lepton Flavor Violation in the Decays $\tau^{ \pm} \rightarrow e^{ \pm} \gamma$ and $\tau^{ \pm} \rightarrow \mu^{ \pm} \gamma$, Phys. Rev. Lett. 104 (2010) 021802 [arXiv:0908.2381] [INSPIRE].

[6] Belle collaboration, K. Hayasaka et al., New search for $\tau \rightarrow e \gamma$ and $\tau \rightarrow \mu \gamma$ decays at Belle, Phys. Lett. B 666 (2008) 16 [arXiv:0705. 0650] [INSPIRE].

[7] MEG collaboration, J. Adam et al., New constraint on the existence of the $\mu^{+} \rightarrow e^{+} \gamma$ decay, Phys. Rev. Lett. 110 (2013) 201801 [arXiv: 1303.0754] [INSPIRE].

[8] K. Hayasaka et al., Search for Lepton Flavor Violating Tau Decays into Three Leptons with 719 Million Produced $\tau^{+} \tau^{-}$Pairs, Phys. Lett. B 687 (2010) 139 [arXiv:1001.3221] [INSPIRE].

[9] SINDRUM collaboration, U. Bellgardt et al., Search for the Decay $\mu^{+} \rightarrow e^{+} e^{+} e^{-}$, Nucl. Phys. B 299 (1988) 1 [INSPIRE].

[10] ACME collaboration, J. Baron et al., Order of Magnitude Smaller Limit on the Electric Dipole Moment of the Electron, Science 343 (2014) 269 [arXiv:1310.7534] [InSPIRE].

[11] Muon (G-2) collaboration, G.W. Bennett et al., An Improved Limit on the Muon Electric Dipole Moment, Phys. Rev. D 80 (2009) 052008 [arXiv:0811.1207] [inSPIRE].

[12] BeLle collaboration, K. Inami et al., Search for the electric dipole moment of the tau lepton, Phys. Lett. B 551 (2003) 16 [hep-ex/0210066] [INSPIRE].

[13] F. Borzumati and A. Masiero, Large Muon and electron Number Violations in Supergravity Theories, Phys. Rev. Lett. 57 (1986) 961 [inSPIRE].

[14] A. Brignole and A. Rossi, Anatomy and phenomenology of mu-tau lepton flavor violation in the MSSM, Nucl. Phys. B 701 (2004) 3 [hep-ph/0404211] [inSPIRE].

[15] P. Paradisi, Constraints on SUSY lepton flavor violation by rare processes, JHEP 10 (2005) 006 [hep-ph/0505046] [INSPIRE].

[16] W. Altmannshofer, A.J. Buras, S. Gori, P. Paradisi and D.M. Straub, Anatomy and Phenomenology of FCNC and CPV Effects in SUSY Theories, Nucl. Phys. B 830 (2010) 17 [arXiv:0909.1333] [INSPIRE].

[17] J. Girrbach, S. Mertens, U. Nierste and S. Wiesenfeldt, Lepton flavour violation in the MSSM, JHEP 05 (2010) 026 [arXiv:0910.2663] [INSPIRE]. 
[18] P. Minkowski, $\mu \rightarrow$ er at a Rate of One Out of 1-Billion Muon Decays?, Phys. Lett. B 67 (1977) 421 [INSPIRE].

[19] A. Ilakovac and A. Pilaftsis, Flavor violating charged lepton decays in seesaw-type models, Nucl. Phys. B 437 (1995) 491 [hep-ph/9403398] [INSPIRE].

[20] J. Hisano, T. Moroi, K. Tobe and M. Yamaguchi, Lepton flavor violation via right-handed neutrino Yukawa couplings in supersymmetric standard model, Phys. Rev. D 53 (1996) 2442 [hep-ph/9510309] [INSPIRE].

[21] J. Hisano and K. Tobe, Neutrino masses, muon g-2 and lepton flavor violation in the supersymmetric seesaw model, Phys. Lett. B 510 (2001) 197 [hep-ph/0102315] [INSPIRE].

[22] K.S. Babu and C. Kolda, Higgs mediated $\tau \rightarrow 3 \mu$ in the supersymmetric seesaw model, Phys. Rev. Lett. 89 (2002) 241802 [hep-ph/0206310] [INSPIRE].

[23] A. Masiero, S.K. Vempati and O. Vives, Massive neutrinos and flavor violation, New J. Phys. 6 (2004) 202 [hep-ph/0407325] [INSPIRE].

[24] A. Dedes, H.E. Haber and J. Rosiek, Seesaw mechanism in the sneutrino sector and its consequences, JHEP 11 (2007) 059 [arXiv:0707.3718] [INSPIRE].

[25] S. Antusch, E. Arganda, M.J. Herrero and A.M. Teixeira, Impact of theta(13) on lepton flavour violating processes within SUSY seesaw, JHEP 11 (2006) 090 [hep-ph/0607263] [INSPIRE].

[26] A. Ilakovac, A. Pilaftsis and L. Popov, Charged lepton flavor violation in supersymmetric low-scale seesaw models, Phys. Rev. D 87 (2013) 053014 [arXiv:1212.5939] [INSPIRE].

[27] A. Ilakovac, A. Pilaftsis and L. Popov, Lepton Dipole Moments in Supersymmetric Low-Scale Seesaw Models, Phys. Rev. D 89 (2014) 015001 [arXiv:1308.3633] [InSPIRE].

[28] A. de Gouvêa, S. Lola and K. Tobe, Lepton flavor violation in supersymmetric models with trilinear R-parity violation, Phys. Rev. D 63 (2001) 035004 [hep-ph/0008085] [INSPIRE].

[29] A. Abada, S. Davidson and M. Losada, Neutrino masses and mixings in the MSSM with soft bilinear R(p) violation, Phys. Rev. D 65 (2002) 075010 [hep-ph/0111332] [INSPIRE].

[30] A. Dedes, S. Rimmer and J. Rosiek, Neutrino masses in the lepton number violating MSSM, JHEP 08 (2006) 005 [hep-ph/0603225] [INSPIRE].

[31] M. Blanke, A.J. Buras, B. Duling, A. Poschenrieder and C. Tarantino, Charged Lepton Flavour Violation and $(\mathrm{g}-2)(\mathrm{mu})$ in the Littlest Higgs Model with T-Parity: A Clear Distinction from Supersymmetry, JHEP 05 (2007) 013 [hep-ph/0702136] [INSPIRE].

[32] S. Kanemura et al., Search for lepton flavor violation in the Higgs boson decay at a linear collider, Phys. Lett. B 599 (2004) 83 [hep-ph/0406316] [InSPIRE].

[33] S. Kanemura, T. Ota and K. Tsumura, Lepton flavor violation in Higgs boson decays under the rare tau decay results, Phys. Rev. D 73 (2006) 016006 [hep-ph/0505191] [INSPIRE].

[34] P. Paradisi, Higgs-mediated $\tau \rightarrow \mu$ and $\tau \rightarrow e$ transitions in II Higgs doublet model and supersymmetry, JHEP 02 (2006) 050 [hep-ph/0508054] [INSPIRE].

[35] A. Crivellin, A. Kokulu and C. Greub, Flavor-phenomenology of two-Higgs-doublet models with generic Yukawa structure, Phys. Rev. D 87 (2013) 094031 [arXiv:1303.5877] [INSPIRE].

[36] A.J. Buras, C. Grojean, S. Pokorski and R. Ziegler, FCNC Effects in a Minimal Theory of Fermion Masses, JHEP 08 (2011) 028 [arXiv:1105.3725] [INSPIRE]. 
[37] G. D'Ambrosio, G.F. Giudice, G. Isidori and A. Strumia, Minimal flavor violation: An Effective field theory approach, Nucl. Phys. B 645 (2002) 155 [hep-ph/0207036] [InSPIRE].

[38] V. Cirigliano, B. Grinstein, G. Isidori and M.B. Wise, Minimal flavor violation in the lepton sector, Nucl. Phys. B 728 (2005) 121 [hep-ph/0507001] [INSPIRE].

[39] E. Nikolidakis and C. Smith, Minimal Flavor Violation, Seesaw and R-parity, Phys. Rev. D 77 (2008) 015021 [arXiv:0710.3129] [inSPIRE].

[40] B.M. Dassinger, T. Feldmann, T. Mannel and S. Turczyk, Model-independent analysis of lepton flavour violating tau decays, JHEP 10 (2007) 039 [arXiv:0707.0988] [INSPIRE].

[41] J.A. Aguilar-Saavedra, A minimal set of top anomalous couplings, Nucl. Phys. B $\mathbf{8 1 2}$ (2009) 181 [arXiv:0811.3842] [INSPIRE].

[42] R. Contino, M. Ghezzi, C. Grojean, M. Muhlleitner and M. Spira, Effective Lagrangian for a light Higgs-like scalar, JHEP 07 (2013) 035 [arXiv: 1303.3876] [INSPIRE].

[43] B. Grzadkowski, Z. Hioki, K. Ohkuma and J. Wudka, Probing anomalous top quark couplings induced by dimension-six operators at photon colliders, Nucl. Phys. B 689 (2004) 108 [hep-ph/0310159] [INSPIRE].

[44] W. Buchmüller and D. Wyler, Effective Lagrangian Analysis of New Interactions and Flavor Conservation, Nucl. Phys. B 268 (1986) 621 [INSPIRE].

[45] B. Grzadkowski, M. Iskrzynski, M. Misiak and J. Rosiek, Dimension-Six Terms in the Standard Model Lagrangian, JHEP 10 (2010) 085 [arXiv: 1008.4884] [INSPIRE].

[46] A. Goudelis, O. Lebedev and J.-h. Park, Higgs-induced lepton flavor violation, Phys. Lett. B 707 (2012) 369 [arXiv:1111.1715] [InSPIRE].

[47] G. Blankenburg, J. Ellis and G. Isidori, Flavour-Changing Decays of a $125 \mathrm{GeV}$ Higgs-like Particle, Phys. Lett. B $\mathbf{7 1 2}$ (2012) 386 [arXiv:1202.5704] [INSPIRE].

[48] R. Harnik, J. Kopp and J. Zupan, Flavor Violating Higgs Decays, JHEP 03 (2013) 026 [arXiv: 1209.1397] [INSPIRE].

[49] M.B. Einhorn and J. Wudka, The Bases of Effective Field Theories, Nucl. Phys. B 876 (2013) 556 [arXiv: 1307.0478] [INSPIRE].

[50] V.A. Smirnov, Asymptotic expansions in limits of large momenta and masses, Commun. Math. Phys. 134 (1990) 109 [INSPIRE].

[51] J. de Blas, M. Chala and J. Santiago, Global Constraints on Lepton-Quark Contact Interactions, Phys. Rev. D 88 (2013) 095011 [arXiv: 1307.5068] [INSPIRE].

[52] M. Cadoret et al., Combination of Bloch oscillations with a Ramsey-Borde interferometer: New determination of the fine structure constant, Phys. Rev. Lett. 101 (2008) 230801 [arXiv:0810.3152] [INSPIRE].

[53] A. Crivellin, J. Girrbach and U. Nierste, Yukawa coupling and anomalous magnetic moment of the muon: an update for the LHC era, Phys. Rev. D 83 (2011) 055009 [arXiv:1010.4485] [INSPIRE].

[54] G.F. Giudice, P. Paradisi and M. Passera, Testing new physics with the electron g-2, JHEP 11 (2012) 113 [arXiv:1208.6583] [INSPIRE].

[55] M. Passera, The Standard model prediction of the muon anomalous magnetic moment, J. Phys. G 31 (2005) R75 [hep-ph/0411168] [INSPIRE]. 
[56] M. Passera, Status of the standard model prediction of the muon g-2, Nucl. Phys. Proc. Suppl. 155 (2006) 365 [hep-ph/0509372] [INSPIRE].

[57] M. Davier, A. Hoecker, B. Malaescu and Z. Zhang, Reevaluation of the Hadronic Contributions to the Muon g-2 and to alpha(MZ), Eur. Phys. J. C 71 (2011) 1515 [Erratum ibid. C 72 (2012) 1874] [arXiv: 1010.4180] [INSPIRE].

[58] K. Hagiwara, R. Liao, A.D. Martin, D. Nomura and T. Teubner, $(g-2)_{\mu}$ and $\alpha\left(M_{Z}^{2}\right)$ re-evaluated using new precise data, J. Phys. G 38 (2011) 085003 [arXiv:1105.3149] [INSPIRE].

[59] T. Blum et al., The Muon (g-2) Theory Value: Present and Future, arXiv:1311.2198 [INSPIRE].

[60] M. Fael, L. Mercolli and M. Passera, Towards a determination of the tau lepton dipole moments, arXiv: 1301.5302 [INSPIRE].

[61] B. Jamieson et al., Measurement of $P(m u)$ xi in polarized muon decay, Phys. Rev. D 74 (2006) 072007 [hep-ex/0605100] [inSPIRE].

[62] J.F. Nieves and P.B. Pal, Generalized Fierz identities, Am. J. Phys. 72 (2004) 1100 [hep-ph/0306087] [INSPIRE].

[63] ILC collaboration, G. Aarons et al., International Linear Collider Reference Design Report Volume 2: Physics at the ILC, arXiv:0709.1893 [INSPIRE].

[64] DELPHI collaboration, P. Abreu et al., Search for lepton flavor number violating $Z^{0}$ decays, Z. Phys. C 73 (1997) 243 [inSPIRE]. 